\title{
The Borsuk-Ulam property for homotopy classes of selfmaps of surfaces of Euler characteristic zero
}

\author{
DACIBERG LIMA GONÇALVES * JOHN GUASCHI ${ }^{\dagger}$ \\ VINICIUS CASTELUBER LAASS $\ddagger$
}

29th July 2016

\begin{abstract}
Let $M$ and $N$ be topological spaces such that $M$ admits a free involution $\tau$. A homotopy class $\beta \in[M, N]$ is said to have the Borsuk-Ulam property with respect to $\tau$ if for every representative map $f: M \rightarrow N$ of $\beta$, there exists a point $x \in M$ such that $f(\tau(x))=f(x)$. In the case where $M$ is a compact, connected manifold without boundary and $N$ is a compact, connected surface without boundary different from the 2 -sphere and the real projective plane, we formulate this property in terms of the pure and full 2-string braid groups of $N$, and of the fundamental groups of $M$ and the orbit space of $M$ with respect to the action of $\tau$. If $M=N$ is either the 2-torus $\mathbb{T}^{2}$ or the Klein bottle $\mathbb{K}^{2}$, we then solve the problem of deciding which homotopy classes of $[M, M]$ have the Borsuk-Ulam property. First, if $\tau: \mathbb{T}^{2} \rightarrow \mathbb{T}^{2}$ is a free involution that preserves orientation, we show that no homotopy class of $\left[\mathbb{T}^{2}, \mathbb{T}^{2}\right]$ has the Borsuk-Ulam property with respect to $\tau$. Secondly, we prove that up to a certain equivalence relation, there is only one class of free involutions $\tau: \mathbb{T}^{2} \rightarrow \mathbb{T}^{2}$ that reverse orientation, and for such involutions, we classify the homotopy classes in $\left[\mathbb{T}^{2}, \mathbb{T}^{2}\right]$ that have the Borsuk-Ulam property with respect to $\tau$ in terms of the induced homomorphism on the fundamental group. Finally, we show that if $\tau: \mathbb{K}^{2} \rightarrow \mathbb{K}^{2}$ is a free involution, then a homotopy class of $\left[\mathbb{K}^{2}, \mathbb{K}^{2}\right]$ has the Borsuk-Ulam property with respect to $\tau$ if and only if the given homotopy class lifts to the torus.
\end{abstract}

\section{Introduction}

In the early twentieth century, St. Ulam conjectured that if $f: \mathbb{S}^{n} \rightarrow \mathbb{R}^{n}$ is a continuous map, there exists $x \in \mathbb{S}^{n}$ such that $f(A(x))=f(x)$, where $A: \mathbb{S}^{n} \rightarrow \mathbb{S}^{n}$ is the antipodal map. The confirmation of this result by K. Borsuk in 1933 [4], known as the Borsuk-Ulam theorem, was the beginning of what we now refer to as Borsuk-Ulam type theorems or the Borsuk-Ulam property. More information about the history and some applications of the Borsuk-Ulam theorem may be found in [13], for example.

One possible generalisation of the classical Borsuk-Ulam theorem is to substitute $\mathbb{S}^{n}$ and $\mathbb{R}^{n}$ by other spaces and to replace the antipodal map by a free involution. A natural question is the following: does every continuous map collapse an orbit of the involution? More precisely, given

*Departamento de Matemática, IME, Universidade de São Paulo, Caixa Postal 66281, Ag. Cidade de São Paulo, CEP: 05314-970, São Paulo, SP, Brazil. e-mail: dlgoncal@ime.usp.br

${ }^{\dagger}$ Normandie Univ., UNICAEN, CNRS, Laboratoire de Mathématiques Nicolas Oresme UMR CNRS 6139, 14000 Caen, France. e-mail: john.guaschi@unicaen.fr

$\ddagger$ Departamento de Matemática, IME, Universidade Federal da Bahia, Av. Adhemar de Barros, S/N Ondina CEP: 40170-110, Salvador, BA, Brazil. e-mail: vinicius.laass@ufba.br

Key words: Borsuk-Ulam theorem, homotopy class, braid groups, surfaces. 
topological spaces $M$ and $N$ such that $M$ admits a free involution $\tau$, we say that the triple $(M, \tau ; N)$ has the Borsuk-Ulam property if for every continuous map $f: M \rightarrow N$, there exists a point $x \in M$ such that $f(\tau(x))=f(x)$. Note that in the whole of this paper, we suppose without further comment that our topological spaces are connected.

In [7], if $M$ is a compact surface without boundary, D. Gonçalves presented a complete description of the triples $\left(M, \tau ; \mathbb{R}^{2}\right)$ that have the Borsuk-Ulam property. In [8], if $M$ and $N$ are compact surfaces without boundary, D. Gonçalves and J. Guaschi described the triples $(M, \tau ; N)$ that have the BorsukUlam property. If the triple $\left(M, \tau ; \mathbb{R}^{2}\right)$ does not have the Borsuk-Ulam property, by definition, there exists a continuous map $f: M \rightarrow \mathbb{R}^{2}$ such that $f(\tau(x)) \neq f(x)$ for all $x \in M$. The fact that there is a single homotopy class of maps from $M$ to $\mathbb{R}^{2}$ implies that if $g: M \rightarrow \mathbb{R}^{2}$ is a continuous map such that $g(\tau(x))=g(x)$ for some $x \in M$, then $g$ is homotopic to $f$. In other words, $g$ is homotopic to a continuous map that does not collapse the orbits of the involution $\tau$.

The situation is different if the triple $(M, \tau ; N)$ does not have the Borsuk-Ulam property and the set $[M, N]$ has cardinality greater than one. Once more, there exists a map $f: M \rightarrow N$ such that $f(\tau(x)) \neq f(x)$ for all $x \in M$. But if $g: M \rightarrow N$ is a continuous map such that $g(\tau(x))=g(x)$ for some $x \in M$, we do not know whether $g$ is homotopic to a map that is injective on each orbit of the involution, unless $g$ is homotopic to $f$. From these observations, we have a natural refinement of the Borsuk-Ulam property, in the following way: we say that a homotopy class $\beta \in[M, N]$ satisfies the Borsuk-Ulam property with respect to $\tau$ if for every map $f: M \rightarrow N$, where $f \in \beta$, there exists $x \in M$ such that $f(\tau(x))=f(x)$. In conjunction with [8], these observations give rise to the following Borsuk-Ulam problem: given compact surfaces $M$ and $N$ without boundary and a free involution $\tau: M \rightarrow M$, classify the elements of the set of homotopy classes $[M, N]$ that have the Borsuk-Ulam property. Before Proposition 9, we recall the definition of an equivalence relation on free involutions. This relation is suitable for the study of this problem.

In this paper, we solve this problem for the cases where $M$ and $N$ coincide and are compact surfaces without boundary of Euler characteristic zero, namely the 2-torus $\mathbb{T}^{2}$ or the Klein bottle $\mathbb{K}^{2}$. We have three main results. First, suppose that $M=\mathbb{T}^{2}$.

Theorem 1. Let $\tau: \mathbb{T}^{2} \rightarrow \mathbb{T}^{2}$ be a free involution that preserves orientation. If $\beta \in\left[\mathbb{T}^{2}, \mathbb{T}^{2}\right]$ is a homotopy class then $\beta$ does not have the Borsuk-Ulam property with respect to $\tau$.

Let us consider free involutions of the torus that reverse orientation. We will show that there is only one equivalence class of such involutions. Let $\tau_{2}: \mathbb{T}^{2} \rightarrow \mathbb{T}^{2}$ be the orientation-reversing involution that admits the lifting to the plane given by $\widehat{\tau}_{2}(x, y)=\left(x+\frac{1}{2}, 1-y\right)$ for all $(x, y) \in \mathbb{R}^{2}$ (see Section 5 ).

Theorem 2. Let $\beta \in\left[\mathbb{T}^{2}, \mathbb{T}^{2}\right]$ be a homotopy class and let $\left(\begin{array}{ll}\beta_{1,1} & \beta_{1,2} \\ \beta_{2,1} & \beta_{2,2}\end{array}\right)$ be the integral matrix of the homomorphism induced by $\beta$ on the fundamental group. Then $\beta$ has the Borsuk-Ulam property with respect to $\tau_{2}$ if and only if $\left(\beta_{1,1}, \beta_{2,1}\right) \neq(0,0)$, and $\beta_{1,2}$ and $\beta_{2,2}$ are both even.

If $\tau_{2}^{\prime}$ is a free involution that reverses orientation it follows from Proposition 25 that $\tau_{2}$ and $\tau_{2}^{\prime}$ are equivalent. In conjunction with Proposition 9 and Theorem 2 this enables us to classify the homotopy classes $\beta$ that satisfy the Borsuk-Ulam property with respect to any such free involution $\tau_{2}^{\prime}$.

Now suppose that the surface under consideration is the Klein bottle $\mathbb{K}^{2}$.

Theorem 3. Let $\tau: \mathbb{K}^{2} \rightarrow \mathbb{K}^{2}$ be a free involution. A homotopy class $\beta \in\left[\mathbb{K}^{2}, \mathbb{K}^{2}\right]$ has the BorsukUlam property with respect to $\tau$ if and only if $\beta$ lifts to the torus.

This papers contains five sections besides the introduction. In Section 2, we first provide an algebraic description of the sets $[M, N]$ and $\left[M, m_{1} ; N, n_{1}\right]$ (the set of pointed homotopy classes) in the case where $M$ and $N$ are manifolds without boundary such that $N$ is a $K(\pi, 1)$ (see Theorem 4 ). 
In Lemma 5, we determine an algebraic criterion to decide whether a given homotopy class contains a representative that is an equivariant map. If $N$ is a compact surface without boundary different from $\mathbb{S}^{2}$ and $\mathbb{R P}^{2}$, in Propositions 6 and 7 we give an algebraic condition to decide whether a homotopy class of maps between $M$ and $N$ has the Borsuk-Ulam property, and in Proposition 10 we show that the set of equivalence classes of free involutions of a compact surface without boundary different from $\mathbb{S}^{2}$ and $\mathbb{R P}^{2}$ is in one-to-one correspondence with the equivalence classes of certain short exact sequences. In Section 3 (resp. Section 4), we study the 2-string braid groups of $M$, where $M=\mathbb{T}^{2}$ (resp. $M=\mathbb{K}^{2}$ ). In Theorem 11 (resp. Theorem [16), we give a presentation of $P_{2}(M)$, from which we deduce in Theorem 14 (resp. Theorem 23 ) that $P_{2}(M)$ is isomorphic to $F(x, y) \oplus \mathbb{Z} \oplus \mathbb{Z}(c f$. [2, Lemma 17]), where $F(x, y)$ is the free group on the set $\{x, y\}$ (resp. to a semi-direct product of the form $F(u, v) \rtimes_{\theta}(\mathbb{Z} \rtimes \mathbb{Z})$ ). In Theorem 14 (resp. Theorem 24), we describe the action by conjugation of the element $\sigma \in B_{2}(M) \backslash P_{2}(M)$ on $P_{2}(M)$. In Section 5, in Theorem 25, we show that up to the above-mentioned equivalence relation, there are precisely two classes of free involutions of the torus that correspond to the orientation-preserving and orientation-reversing involutions respectively, and we develop some results and arguments necessary to prove Theorem 1 and 2, Finally, in Section 6, we show in Theorem 33 that up to the above-mentioned equivalence relation, there is just one class of free involutions of the Klein bottle, and we prove Theorem 3 ,

\section{Preliminaries and Generalities}

Let $\left(M, m_{1}\right)$ and $\left(N, n_{1}\right)$ be pointed manifolds, and suppose that $\pi_{i}\left(N, n_{1}\right)$ is trivial for all $i \geq 2$. Let $[M, N]$ (resp. $\left.\left[M, m_{1} ; N, n_{1}\right]\right)$ denote the set of free (resp. pointed) homotopy class of maps from $M$ to $N$ (resp. from $\left(M, m_{1}\right)$ to $\left.\left(N, n_{1}\right)\right)$, and let $\operatorname{Hom}\left(\pi_{1}\left(M, m_{1}\right), \pi_{1}\left(N, n_{1}\right)\right)$ denote the set of homomorphisms between the fundamental groups of $M$ and $N$. If $f, g:\left(M, m_{1}\right) \rightarrow\left(N, n_{1}\right)$ are homotopic pointed maps, denoted by $f \simeq g$ (rel. $m_{1}$ ), then the induced homomorphisms $f_{\#}, g_{\#}$ : $\pi_{1}\left(M, m_{1}\right) \rightarrow \pi_{1}\left(N, n_{1}\right)$ on the level of fundamental groups are equal. Given a pointed homotopy class $\alpha \in\left[M, m_{1} ; N, n_{1}\right]$, we may thus associate a homomorphism $\alpha_{\#} \in \operatorname{Hom}\left(\pi_{1}\left(M, m_{1}\right), \pi_{1}\left(N, n_{1}\right)\right)$ by choosing a representative map $f:\left(M, m_{1}\right) \rightarrow\left(N, n_{1}\right)$ of $\alpha$, and by taking the induced homomorphism. This gives rise to the following well-defined map:

$$
\begin{aligned}
\Gamma_{M, N}:\left[M, m_{1} ; N, n_{1}\right] & \longrightarrow \operatorname{Hom}\left(\pi_{1}\left(M, m_{1}\right), \pi_{1}\left(N, n_{1}\right)\right) \\
\alpha=[f] & \longmapsto \alpha_{\#}:=f_{\#} \cdot
\end{aligned}
$$

It is well known that the map $\Gamma_{M, N}$ is a bijection [17, Chapter V, Theorem 4.3]. If $f, g:\left(M, m_{1}\right) \rightarrow$ $\left(N, n_{1}\right)$ are pointed maps such that $f \simeq g$ (rel. $\left.m_{1}\right)$, then omitting the base points, the maps $f, g: M \rightarrow N$ are homotopic. Given a pointed homotopy class $\alpha \in\left[M, m_{1} ; N, n_{1}\right]$, we may thus associate a free homotopy class $\alpha_{\mathcal{F}} \in[M, N]$ by choosing a representative map $f:\left(M, m_{1}\right) \rightarrow\left(N, n_{1}\right)$ of $\alpha$, and by taking the free homotopy class $\alpha_{\mathcal{F}}$ that represents the map $f: M \rightarrow N$, from which we obtain the following well-defined map:

$$
\begin{aligned}
\Lambda_{M, N}:\left[M, m_{1} ; N, n_{1}\right] & \longrightarrow[M, N] \\
\alpha=[f] & \longmapsto \alpha_{\mathcal{F}}:=[f],
\end{aligned}
$$

that is surjective by [16, Lemma 6.4].

Two homomorphisms $h_{1}, h_{2} \in \operatorname{Hom}\left(\pi_{1}\left(M, m_{1}\right), \pi_{1}\left(N, n_{1}\right)\right)$ are said to be equivalent, written $h_{1} \sim h_{2}$, if there exists $\omega \in \pi_{1}\left(N, n_{1}\right)$ such that $h_{1}(v)=\omega h_{2}(v) \omega^{-1}$ for all $v \in \pi_{1}\left(M, m_{1}\right)$. It is straightforward to see that $\sim$ is an equivalence relation. The associated canonical projection shall be denoted as follows:

$$
\Upsilon_{M, N}: \operatorname{Hom}\left(\pi_{1}\left(M, m_{1}\right), \pi_{1}\left(N, n_{1}\right)\right) \longrightarrow \frac{\operatorname{Hom}\left(\pi_{1}\left(M, m_{1}\right), \pi_{1}\left(N, n_{1}\right)\right)}{\sim} .
$$


By [17, Chapter V, Corollary 4.4], there exists a bijective map:

$$
\Delta_{M, N}:[M, N] \longrightarrow \frac{\operatorname{Hom}\left(\pi_{1}\left(M, m_{1}\right), \pi_{1}\left(Y, n_{1}\right)\right)}{\sim}
$$

such that $\Delta_{M, N} \circ \Lambda_{M, N}=\Upsilon_{M, N} \circ \Gamma_{M, N}$. Given an element $\beta \in[M, N]$, we denote the equivalence class $\Delta_{M, N}(\beta)$ by $\beta_{\#}$. We sum up these observations in the following theorem, that we shall often use in this paper.

Theorem 4. If $\left(M, m_{1}\right)$ and $\left(N, n_{1}\right)$ are pointed manifolds such that $\pi_{i}\left(N, n_{1}\right)$ is trivial for all $i \geq 2$, then the following diagram is commutative:

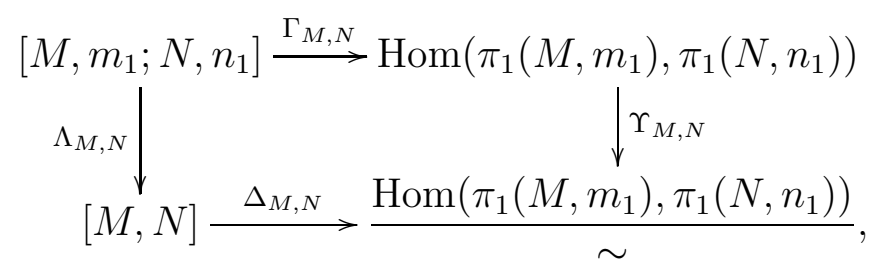

where the horizontal arrows are bijections, and the vertical arrows are surjective.

Let $M$ be a manifold, and let $\tau: M \rightarrow M$ be a free involution. Let $M_{\tau}$ denote the corresponding orbit space, which is also a manifold, and let $p_{\tau}: M \rightarrow M_{\tau}$ denote the associated double covering. This gives rise to the following short exact sequence:

$$
1 \longrightarrow \pi_{1}\left(M, m_{1}\right) \stackrel{\left(p_{\tau}\right)_{\#}}{\longrightarrow} \pi_{1}\left(M_{\tau}, p_{\tau}\left(m_{1}\right)\right) \stackrel{\theta_{\tau}}{\longrightarrow} \mathbb{Z}_{2} \longrightarrow 1
$$

that we call the short exact sequence induced by $\tau$, and that we denote by $S_{\tau}$, where we identify $\frac{\pi_{1}\left(M_{\tau}, p_{\tau}\left(m_{1}\right)\right)}{\left(p_{\tau}\right)_{\#}\left(\pi_{1}\left(M, m_{1}\right)\right)}$ with $\mathbb{Z}_{2}=\{\overline{0}, \overline{1}\}$, and $\theta_{\tau}$ is the natural projection onto the quotient.

We now prove an algebraic criterion to decide whether a pointed homotopy class has an equivariant representative map, which will help in simplifying the proofs of Propositions 6 and 10 .

Lemma 5. Let $\left(M, m_{1}\right)$ and $\left(N, n_{1}\right)$ be pointed manifolds (resp. compact surfaces without boundary) such that $\pi_{i}\left(N, n_{1}\right)$ is trivial for all $i \geq 2$, let $\tau: M \rightarrow M$ and $\tau_{1}: N \rightarrow N$ be free involutions. Given a pointed homotopy class $\alpha \in\left[M, m_{1} ; N, n_{1}\right]$, the following conditions are equivalent:

1. there exists a representative map (resp. homeomorphism) $f:\left(M, m_{1}\right) \rightarrow\left(N, n_{1}\right)$ of $\alpha$ that is $\left(\tau, \tau_{1}\right)$-equivariant, i.e. $f(\tau(x))=\tau_{1}(f(x))$ for all $x \in M$.

2. there exists a homomorphism (resp. isomorphism) $\psi: \pi_{1}\left(M_{\tau}, p_{\tau}\left(m_{1}\right)\right) \rightarrow \pi_{1}\left(N_{\tau_{1}}, p_{\tau_{1}}\left(n_{1}\right)\right)$ such that the following diagram is commutative:

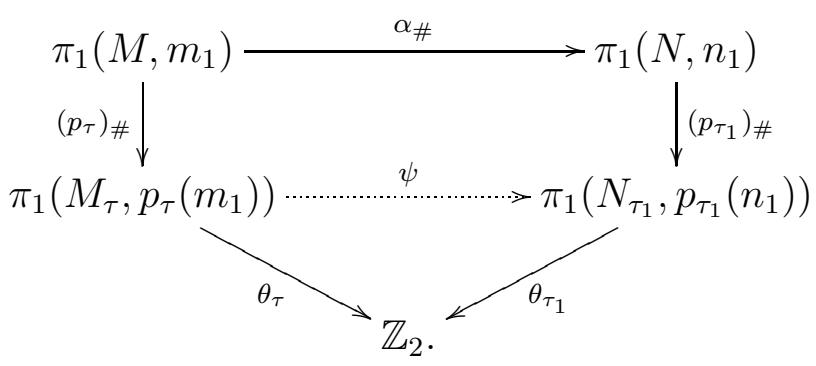

Proof. $(1 \Rightarrow 2)$ Suppose first that $f:\left(M, m_{1}\right) \rightarrow\left(N, n_{1}\right)$ is a representative map of $\alpha$ that is $\left(\tau, \tau_{1}\right)$ equivariant. Then $f\{x, \tau(x)\}=\left\{f(x), \tau_{1}(f(x))\right\}$ for all $x \in M$, and hence the map $f$ induces a map of 
the corresponding orbit spaces, in other words, there exists a map $\bar{f}:\left(M_{\tau}, p_{\tau}\left(m_{1}\right)\right) \rightarrow\left(N_{\tau_{1}}, p_{\tau_{1}}\left(n_{1}\right)\right)$ such that the following diagram is commutative:

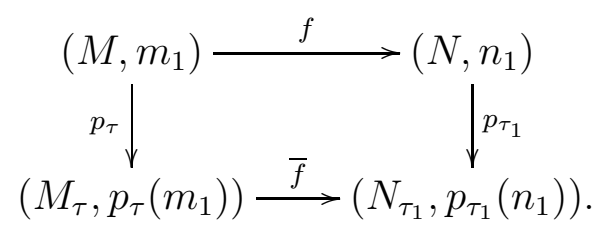

Diagram (7) implies that $\left(p_{\tau_{1}}\right)_{\#} \circ \alpha_{\#}=\psi \circ\left(p_{\tau}\right)_{\#}$, and also implies that $\theta_{\tau_{1}} \circ \psi=\theta_{\tau}$ using standard covering space arguments and the fact that the map $f$ is equivariant. We thus obtain the commutative diagram (6) . Further, if $f$ is a homeomorphism, then $\bar{f}$ is too, and so the induced homomorphism $\psi=\bar{f}_{\#}: \pi_{1}\left(M_{\tau}, p_{\tau}\left(m_{1}\right)\right) \rightarrow \pi_{1}\left(N_{\tau_{1}}, p_{\tau_{1}}\left(n_{1}\right)\right)$ is an isomorphism.

$(2 \Rightarrow 1)$ Suppose that there exists a homomorphism $\psi: \pi_{1}\left(M_{\tau}, p_{\tau}\left(m_{1}\right)\right) \rightarrow \pi_{1}\left(N_{\tau_{1}}, p_{\tau_{1}}\left(n_{1}\right)\right)$ for which diagram (6) is commutative. Since $p_{\tau_{1}}: N \rightarrow N_{\tau_{1}}$ is a covering map, the triviality of $\pi_{i}\left(N, n_{1}\right)$ implies that of $\pi_{i}\left(N_{\tau_{1}}, p_{\tau_{1}}\left(n_{1}\right)\right)$ for all $i \geq 2$. It follows from Theorem 4 that there exists a map $\bar{f}:\left(M_{\tau}, p_{\tau}\left(m_{1}\right)\right) \rightarrow\left(N_{\tau_{1}}, p_{\tau_{1}}\left(n_{1}\right)\right)$ such that $\bar{f}_{\#}=\psi$, and so by (6) $)$, there exists a map $f:\left(M, m_{1}\right) \rightarrow$ $\left(N, n_{1}\right)$ that is a lift of the map $\bar{f} \circ p_{\tau}$ for the covering $p_{\tau_{1}}$, so that we have the commutative diagram (17). Using the short exact sequences induced by $\tau$ and $\tau_{1}$ in the sense of (15), one sees that $\alpha_{\#}=f_{\#}$ and so by Theorem $4, f$ is a representative map of $\alpha$. We claim that $f$ is $\left(\tau, \tau_{1}\right)$-equivariant. To do so, note that for all $x \in M$, we have:

$$
\left(p_{\tau_{1}} \circ f \circ \tau\right)(x)=\left(\bar{f} \circ p_{\tau} \circ \tau\right)(x)=\left(\bar{f} \circ p_{\tau}\right)(x)=\left(p_{\tau_{1}} \circ f\right)(x) .
$$

Hence either $f(\tau(x))=f(x)$, or $f(\tau(x))=\tau_{1}(f(x))$. Let $\xi:[0,1] \rightarrow M$ be an arc from $m_{1}$ to $\tau\left(m_{1}\right)$. Then the loop $\gamma=p_{\tau} \circ \xi$ satisfies $\theta_{\tau}([\gamma])=\overline{1}$. By (66) we have:

$$
\theta_{\tau_{1}}([\bar{f} \circ \gamma])=\theta_{\tau_{1}} \circ \bar{f}_{\#}([\gamma])=\theta_{\tau}([\gamma])=\overline{1},
$$

and since $f \circ \xi$ is a lift of the loop $\bar{f} \circ \gamma$ by the covering $p_{\tau_{1}}$, it is an arc that is not a loop. Therefore $f\left(m_{1}\right)=(f \circ \xi)(0) \neq(f \circ \xi)(1)=f\left(\tau\left(m_{1}\right)\right)$, and so $f\left(\tau\left(m_{1}\right)\right)=\tau_{1}\left(f\left(m_{1}\right)\right)$. Using standard covering space arguments and the hypothesis that $N$ is connected, it follows in a straightforward manner that the equality $f(\tau(x))=\tau_{1}(f(x))$ holds for all $x \in M$. This proves the claim. Finally, if $M$ and $N$ are compact surfaces without boundary and $\psi$ is an isomorphism, it follows from the classification theorem for surfaces and [18, Theorem 5.6.2] that $\psi$ is induced by a homeomorphism. So without loss of generality, we may take $\bar{f}$ to be a homeomorphism, and thus $f$ is also a homeomorphism.

Let $N$ be a compact surface without boundary with base point $n_{1} \in N$. Recall that $F_{2}(N)=$ $\left\{\left(y_{1}, y_{2}\right) \in N \times N \mid y_{1} \neq y_{2}\right\}$ is the $2^{\text {nd }}$ configuration space of $N$, and if $\tau_{1}: F_{2}(N) \rightarrow F_{2}(N)$ is the involution defined by $\tau_{1}\left(x_{1}, x_{2}\right)=\left(x_{2}, x_{1}\right)$, then $D_{2}(N)$ is the associated orbit space. Let $n_{2} \in N-\left\{n_{1}\right\}$, and let $n=\left(n_{1}, n_{2}\right) \in F_{2}(N)$. By [6, Corollary 2.2], if $N$ is different from $\mathbb{S}^{2}$ and $\mathbb{R P}^{2}, F_{2}(N)$ and $D_{2}(N)$ are manifolds for which $\pi_{i}\left(F_{2}(N), n\right)$ and $\pi_{i}\left(D_{2}(N), p_{\tau_{1}}(n)\right)$ are trivial for all $i \geq 2$. The groups $P_{2}(N)=\pi_{1}\left(F_{2}(N), n\right)$ and $B_{2}(N)=\pi_{1}\left(D_{2}(N), p_{\tau_{1}}\left(n_{1}\right)\right)$ are the pure and full 2 -string braid groups of $N$ respectively, related by the following short exact sequence:

$$
1 \longrightarrow P_{2}(N) \stackrel{\iota}{\longrightarrow} B_{2}(N) \stackrel{\pi}{\longrightarrow} \mathbb{Z}_{2} \longrightarrow 1
$$

where $\iota=\left(p_{\tau_{1}}\right)_{\#}$ and $\pi=\theta_{\tau_{1}}$.

The following result generalises [8, Proposition 13] and gives an algebraic criterion in terms of braid groups to decide whether a pointed homotopy class has the Borsuk-Ulam property.

Proposition 6. Let $\left(M, m_{1}\right)$ be a pointed manifold, let $\tau: M \rightarrow M$ be a free involution, and let $\left(N, n_{1}\right)$ be a compact surface without boundary different from $\mathbb{S}^{2}$ and $\mathbb{R P}^{2}$. For a pointed homotopy class $\alpha \in\left[M, m_{1} ; N, n_{1}\right]$, the following conditions are equivalent: 
1. $\alpha$ does not have the Borsuk-Ulam property with respect to $\tau$.

2. there exist homomorphisms $\varphi: \pi_{1}\left(M, m_{1}\right) \rightarrow P_{2}(N)$ and $\psi: \pi_{1}\left(M_{\tau}, p_{\tau}\left(m_{1}\right)\right) \rightarrow B_{2}(N)$ for which the following diagram is commutative:

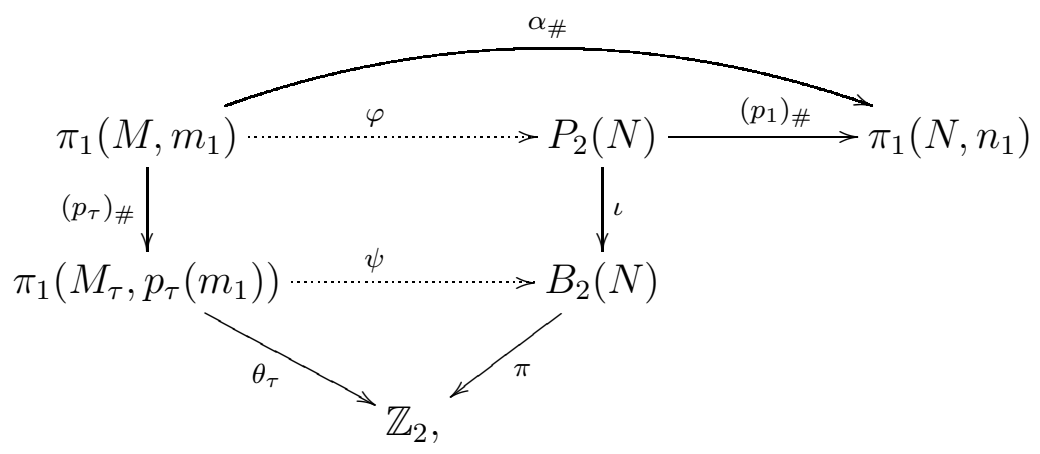

where $p_{1}: F_{2}(N) \rightarrow N$ is projection onto the first coordinate.

Proof. $(1 \Rightarrow 2)$ If $\alpha$ does not have the Borsuk-Ulam property, there exists a map $f:\left(M, m_{1}\right) \rightarrow$ $\left(N, n_{1}\right)$ such that $\alpha=[f]$ and $f(\tau(x)) \neq f(x)$ for all $x \in M$. Hence the map $F: M \rightarrow F_{2}(N)$ given by $F(x)=(f(x), f(\tau(x)))$ is well defined, $\left(\tau, \tau_{1}\right)$-equivariant and satisfies $p_{1} \circ F=f$. Let $\varphi: \pi_{1}\left(M, m_{1}\right) \rightarrow P_{2}(N)$ be the homomorphism induced by $F$. So, we have $\left(p_{1}\right)_{\#} \circ \varphi=\left(p_{1}\right)_{\#} \circ$ $F_{\#}=\left(p_{1} \circ F\right)_{\#}=f_{\#}=\alpha_{\#}$. By Theorem 4 and Lemma 5, there exists a homomorphism $\psi$ : $\pi_{1}\left(M_{\tau}, p_{\tau}\left(m_{1}\right)\right) \rightarrow B_{2}(N)$ such that $\psi \circ\left(p_{\tau}\right)_{\#}=\iota \circ \varphi$ and $\pi \circ \psi=\theta_{\tau}$. This completes the first part of the proof.

$(2 \Rightarrow 1)$ Suppose that diagram (9) is commutative. Recall that $F_{2}(N)$ is a manifold which is a $K(\pi, 1)$. So, Theorem 4 and Lemma 5 imply that the homomorphism $\varphi$ is induced by a $\left(\tau, \tau_{1}\right)$ equivariant map $F: M \rightarrow F_{2}(N)$. Let $f, g: M \rightarrow N$ be maps such that $F(x)=(f(x), g(x))$ for all $x \in M$. Since $F$ is equivariant, we have $f(\tau(x))=g(x) \neq f(x)$ for all $x \in M$. Again, by (9) and Theorem 4 we have $\alpha=[f]$, and thus $\alpha$ does not have the Borsuk-Ulam property.

The following result shows that to solve the Borsuk-Ulam problem for free homotopy classes, it suffices to solve it for pointed homotopy classes.

Proposition 7. Suppose that the hypotheses of Proposition 6 hold.

1. Let $\alpha, \alpha^{\prime} \in\left[M, m_{1} ; N, n_{1}\right]$ and suppose that the homomorphisms $\alpha_{\#}, \alpha_{\#}^{\prime}: \pi_{1}\left(M, m_{1}\right) \rightarrow \pi_{1}\left(N, n_{1}\right)$ are equivalent. Then $\alpha$ does not have the Borsuk-Ulam property with respect to $\tau$ if and only if $\alpha^{\prime}$ does not have the Borsuk-Ulam property with respect to $\tau$.

2. Let $\beta \in[M, N]$. Then there exists $\alpha \in\left[M, m_{1} ; N, n_{1}\right]$ such that $\beta=\alpha_{\mathcal{F}}$. Further, $\beta$ does not have the Borsuk-Ulam property with respect to $\tau$ if and only if $\alpha$ does not have the Borsuk-Ulam property with respect to $\tau$.

Proof. To prove part 1, first note that by the symmetry of the statement with respect to $\alpha$ and $\alpha^{\prime}$, it suffices to prove one of the implications of the conclusion. So suppose that $\alpha$ does not have the Borsuk-Ulam property with respect to $\tau$. By Proposition 6, there exist homomorphisms $\varphi$ : $\pi_{1}\left(M, m_{1}\right) \rightarrow P_{2}(N)$ and $\psi: \pi_{1}\left(M_{\tau}, p_{\tau}\left(m_{1}\right)\right) \rightarrow B_{2}(N)$ such that diagram (9) is commutative. Since the homomorphism $\left(p_{1}\right)_{\#}: P_{2}(N) \rightarrow \pi_{1}\left(N, n_{1}\right)$ is surjective by [3, Theorem 1.4], and the homomorphisms $\alpha_{\#}$ and $\alpha_{\#}^{\prime}$ are equivalent via an element of $\pi_{1}\left(N, n_{1}\right), \gamma$ say, there exists $b \in P_{2}(N)$ such that $\left(p_{1}\right)_{\#}(b)=\gamma$. If $\varphi^{\prime}: \pi_{1}\left(M, m_{1}\right) \rightarrow P_{2}(N)$ (resp. $\left.\psi^{\prime}: \pi_{1}\left(M_{\tau}, p_{\tau}\left(m_{1}\right)\right) \rightarrow B_{2}(N)\right)$ is the homomorphism given by $\varphi^{\prime}(v)=b \varphi(v) b^{-1}$ for all $v \in \pi_{1}\left(M, m_{1}\right)$ (resp. $\psi^{\prime}(w)=\iota(b) \psi(w) \iota(b)^{-1}$ for all $\left.w \in \pi_{1}\left(M_{\tau}, p_{\tau}\left(m_{1}\right)\right)\right)$ where $\iota$ is as in (8)), then diagram (9) remains commutative if we replace $\alpha_{\#}, \varphi$ and $\psi$ by $\alpha_{\#}^{\prime}, \varphi^{\prime}$ and $\psi^{\prime}$. It follows from Proposition 6 that $\alpha^{\prime}$ does not have the Borsuk-Ulam property with respect to $\tau$. 
To prove part 2 , if $\beta \in[M, N]$, by Theorem 4 there exists $\alpha \in\left[M, m_{1} ; N, n_{1}\right]$ such that $\beta=\alpha_{\mathcal{F}}$. Suppose that $\beta$ does not have the Borsuk-Ulam property with respect to $\tau$. So there exists a map $f: M \rightarrow N$ such that $\beta=[f]$ and $f(\tau(x)) \neq f(x)$ for all $x \in M$. By [16, Lemma 6.4], there exists a homeomorphism $H: N \rightarrow N$ that is homotopic to the identity such that $H\left(f\left(m_{1}\right)\right)=n_{1}$. Thus the pointed homotopy class $\alpha^{\prime}=[H \circ f] \in\left[M, m_{1} ; N, n_{1}\right]$ does not have the Borsuk-Ulam property with respect to $\tau$. By equations (1) and (2) and Theorem 4 it follows that:

$$
\begin{aligned}
\Upsilon_{M, N}\left(\alpha_{\#}\right) & =\left(\Upsilon_{M, N} \circ \Gamma_{M, N}\right)(\alpha)=\left(\Delta_{M, N} \circ \Lambda_{M, N}\right)(\alpha)=\Delta_{M, N}\left(\alpha_{\mathcal{F}}\right) \\
& =\Delta_{M, N}(\beta)=\Delta_{M, N}([f])=\Delta_{M, N}([H \circ f])=\Delta_{M, N}\left(\left(\alpha^{\prime}\right)_{\mathcal{F}}\right) \\
& =\left(\Delta_{M, N} \circ \Lambda_{M, N}\right)\left(\alpha^{\prime}\right)=\left(\Upsilon_{M, N} \circ \Gamma_{M, N}\right)\left(\alpha^{\prime}\right)=\Upsilon_{M, N}\left(\alpha_{\#}^{\prime}\right) .
\end{aligned}
$$

Hence the homomorphisms $\alpha_{\#}$ and $\alpha_{\#}^{\prime}$ are equivalent by (3) . We conclude from part 1 that $\alpha$ does not have the Borsuk-Ulam property with respect to $\tau$. The converse is obvious.

Remark 8. Taken together, Theorem 4 and Propositions 6 and 7 provide an algebraic criterion to solve the Borsuk-Ulam problem for free homotopy classes.

To end this section, we discuss briefly which involutions of compact surfaces we should consider in order to solve the Borsuk-Ulam problem for homotopy classes. As in [9, Corollary 2.3] if $\tau_{1}, \tau_{2}$ : $M \rightarrow M$ are free involutions, we say that $\tau_{1}$ and $\tau_{2}$ are equivalent, written $\tau_{1} \sim \tau_{2}$, if there exists a $\left(\tau_{1}, \tau_{2}\right)$-equivariant homeomorphism $H: M \rightarrow M$.

Proposition 9. Let $M$ and $N$ be topological spaces, and let $\tau_{1}, \tau_{2}: M \rightarrow M$ be equivalent free involutions. Let $H: M \rightarrow M$ be a $\left(\tau_{1}, \tau_{2}\right)$-equivariant homeomorphism. Then the map $\mathcal{H}:[M, N] \rightarrow$ $[M, N]$ defined by $\mathcal{H}([f])=\left[f \circ H^{-1}\right]$ is a bijection. Further, a homotopy class $\beta \in[M, N]$ does not have the Borsuk-Ulam property with respect to $\tau_{1}$ if and only if $\mathcal{H}(\beta)$ does not have the Borsuk-Ulam property with respect to $\tau_{2}$.

Proof. Let $\tau_{1}, \tau_{2}: M \rightarrow M$ be free involutions, and suppose that $H: M \rightarrow M$ is a $\left(\tau_{1}, \tau_{2}\right)$-equivariant homeomorphism and that $\beta \in[M, N]$. If $\beta$ does not have the Borsuk-Ulam property with respect to $\tau_{1}$, there exists a map $f_{1}: M \rightarrow N$ such that $\left[f_{1}\right]=\beta$ and $f_{1}\left(\tau_{1}(x)\right) \neq f_{1}(x)$ for all $x \in M$. So the map $f_{2}=f_{1} \circ H^{-1}: M \rightarrow N$ satisfies $\left[f_{2}\right]=\mathcal{H}(\beta)$ and $f_{2}\left(\tau_{2}(x)\right) \neq f_{2}(x)$ for all $x \in M$, in other words $\mathcal{H}(\beta)$ does not have the Borsuk-Ulam property with respect to $\tau_{2}$. The converse follows in a similar manner.

Now let:

$I=\left\{\tau: M \rightarrow M \mid \tau\right.$ is a free involution, $M$ is a compact surface without boundary, $\left.M \neq \mathbb{S}^{2}, \mathbb{R P}^{2}\right\}$,

and let $\mathcal{I}=I / \sim$ be the corresponding quotient set. We give an algebraic interpretation of this definition. Let $E$ denote the set of all short exact sequences of the form $1 \rightarrow A \stackrel{i}{\rightarrow} B \stackrel{j}{\rightarrow} \mathbb{Z}_{2} \rightarrow 1$, where $A$ and $B$ are fundamental groups of compact surfaces without boundary different from $\mathbb{S}^{2}$ and $\mathbb{R P}^{2}$. If $k \in\{1,2\}$, and $S_{k}$ is an element of $E$ of the form $1 \rightarrow A_{k} \stackrel{i_{k}}{\rightarrow} B_{k} \stackrel{j_{k}}{\rightarrow} \mathbb{Z}_{2} \rightarrow 1$, we say that $S_{1}$ and $S_{2}$ are equivalent, written $S_{1} \approx S_{2}$, if there exist isomorphisms $\varphi: A_{1} \rightarrow A_{2}$ and $\psi: B_{1} \rightarrow B_{2}$ such that the following diagram is commutative:

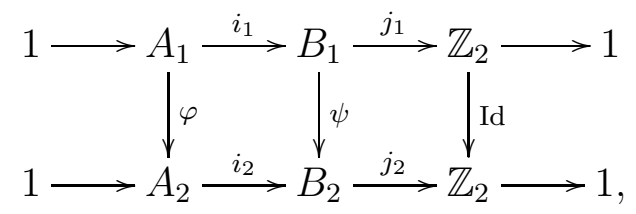

where Id denotes the identity. It is easy to see that $\approx$ is a equivalence relation on $E$. Let $\mathcal{E}=E / \approx$ be the corresponding quotient set. Then we have the following proposition. 
Proposition 10. The map $I \longrightarrow E$ given by (5) that to a free involution $\tau$ associates the short exact sequence $S_{\tau}$ gives rise to a bijection $\Sigma: \mathcal{I} \rightarrow \mathcal{E}$.

Proof. By Lemma 5, $\Sigma$ is well defined and injective. It remains to show that $\Sigma$ is surjective. Let $S \in E$ be a short exact sequence of the form $1 \rightarrow A \stackrel{i}{\rightarrow} B \stackrel{j}{\rightarrow} \mathbb{Z}_{2} \rightarrow 1$, let $M$ and $N$ be compact surfaces without boundary such that $A=\pi_{1}(M)$ and $B=\pi_{1}(N)$, and let $\hat{N}$ be the double covering of $N$ determined by the subgroup $A \subset B$. Since $M$ and $\hat{N}$ have isomorphic fundamental groups, there exists a homeomorphism $h: M \rightarrow \hat{N}$ that realises a given isomorphism between the fundamental groups of $M$ and $\hat{N}$. Then the composition of $h$ with the double covering $\hat{N} \rightarrow N$ is a double covering of $N$ that determines a free involution $\tau$ on $M$, and $\Sigma[\tau]=[S]$ as required.

\section{The braid groups of the torus}

The aim of this section is to prove Theorem 14 that provides an explicit isomorphism between $P_{2}\left(\mathbb{T}^{2}\right)$ and $F(x, y) \oplus \mathbb{Z} \oplus \mathbb{Z}$ and describes the action of $\sigma$ on $P_{2}\left(\mathbb{T}^{2}\right)$.

If $G$ is a group and $a, b \in G$, let $[a, b]=a b a^{-1} b^{-1}$ denote their commutator. The following presentation of $P_{2}\left(\mathbb{T}^{2}\right)$ may be found in [5, Section 4].

Theorem 11. The following is a presentation of $P_{2}\left(\mathbb{T}^{2}\right)$ :

generators: $\rho_{1,1}, \rho_{1,2}, \rho_{2,1}, \rho_{2,2}, B$.

relations:

(i) $\left[\rho_{1,1}, \rho_{1,2}^{-1}\right]=\left[\rho_{2,1}, \rho_{2,2}^{-1}\right]=B$.

(ii) $\rho_{2, k} \rho_{1, k} \rho_{2, k}^{-1}=B \rho_{1, k} B^{-1}$ and $\rho_{2, k}^{-1} \rho_{1, k} \rho_{2, k}=\rho_{1, k}\left[B^{-1}, \rho_{1, k}\right]$ for all $k \in\{1,2\}$.

(iii) $\rho_{2,1} \rho_{1,2} \rho_{2,1}^{-1}=B \rho_{1,2}\left[\rho_{1,1}^{-1}, B\right]$ and $\rho_{2,1}^{-1} \rho_{1,2} \rho_{2,1}=B^{-1}\left[B, \rho_{1,1}\right] \rho_{1,2}\left[B^{-1}, \rho_{1,1}\right]$.

(iv) $\rho_{2,2} \rho_{1,1} \rho_{2,2}^{-1}=\rho_{1,1} B^{-1}$ and $\rho_{2,2}^{-1} \rho_{1,1} \rho_{2,2}=\rho_{1,1} B\left[B^{-1}, \rho_{1,2}\right]$.

Note that $B$ may be removed from the list of generators using relation (ii). In order to obtain a presentation of $P_{2}\left(\mathbb{T}^{2}\right)$ that is more suitable for our purposes, we shall use the following lemma.

Lemma 12. With respect to the presentation of $P_{2}\left(\mathbb{T}^{2}\right)$ given in Theorem 11 , the following relations hold:

(v) $\rho_{2, k} B \rho_{2, k}^{-1}=B \rho_{1, k}^{-1} B \rho_{1, k} B^{-1}$ for all $k \in\{1,2\}$.

(vi) $\rho_{1, k} B^{-1} \rho_{2, k} \rho_{i, j} \rho_{2, k}^{-1} B \rho_{1, k}^{-1}=\rho_{i, j}$ for all $i, j, k \in\{1,2\}$.

Observe that relation (vi) implies that for $k \in\{1,2\}, \rho_{1, k} B^{-1} \rho_{2, k}$ belongs to the centre of $P_{2}\left(\mathbb{T}^{2}\right)$.

Proof of Lemma 12. We have:

$$
\begin{aligned}
& \rho_{2,1} B \rho_{2,1}^{-1} \stackrel{\text { (i) }}{=}\left[\rho_{2,1} \rho_{1,1} \rho_{2,1}^{-1}, \rho_{2,1} \rho_{1,2}^{-1} \rho_{2,1}^{-1} \stackrel{\text { (iii), (iii) }}{=} \rho_{1,1} \rho_{1,2}^{-1} \rho_{1,1}^{-1} \rho_{1,2} \rho_{1,1}^{-1} B \rho_{1,1} B^{-1} \stackrel{\text { (1) }}{=} B \rho_{1,1}^{-1} B \rho_{1,1} B^{-1}\right. \text {, and } \\
& \rho_{2,2} B \rho_{2,2}^{-1} \stackrel{\text { (i) })}{=}\left[\rho_{2,2} \rho_{1,1} \rho_{2,2}^{-1}, \rho_{2,2} \rho_{1,2}^{-1} \rho_{2,2}^{-1}\right] \stackrel{\text { (iii),(iv) }}{=} \rho_{1,1} \rho_{1,2}^{-1} \rho_{1,1}^{-1} B \rho_{1,2} B^{-1} \stackrel{\text { (i) }}{=} B \rho_{1,2}^{-1} B \rho_{1,2} B^{-1} \text {, }
\end{aligned}
$$

which proves ( (v) . To prove (vi) , first let $i=1$. If $j=k$, the relation follows directly from (ii)). If $j \neq k$, we have:

$$
\begin{aligned}
& \rho_{1,2} B^{-1} \rho_{2,2} \rho_{1,1} \rho_{2,2}^{-1} B \rho_{1,2}^{-1} \stackrel{\text { (iv) }}{=} \rho_{1,2} B^{-1} \rho_{1,1} \rho_{1,2}^{-1} \stackrel{\text { (i) }}{=} \rho_{1,1} \text { and } \\
& \rho_{1,1} B^{-1} \rho_{2,1} \rho_{1,2} \rho_{2,1}^{-1} B \rho_{1,1}^{-1} \stackrel{\text { (iii) }}{=} \rho_{1,1} \rho_{1,2} \rho_{1,1}^{-1} B \stackrel{\text { (i) }}{=} \rho_{1,2} .
\end{aligned}
$$


Suppose that $i=2$. If $j=k$ then:

$$
\rho_{1, k} B^{-1} \rho_{2, k} \rho_{2, k} \rho_{2, k}^{-1} B \rho_{1, k}^{-1}=\rho_{1, k} B^{-1} \rho_{2, k} B \rho_{1, k}^{-1} \rho_{2, k}^{-1} \rho_{2, k} \stackrel{(\mathrm{iii)},(\mathbb{V})}{=} \rho_{2, k} .
$$

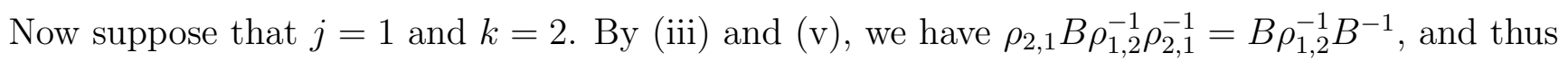
$\rho_{1,2} B^{-1} \rho_{2,2} \rho_{2,1} \rho_{2,2}^{-1} B \rho_{1,2}^{-1}=\rho_{1,2} B^{-1} \rho_{2,2} \rho_{2,1} \rho_{2,2}^{-1} \rho_{2,1}^{-1} B \rho_{1,2}^{-1} B^{-1} \rho_{2,1} \stackrel{\text { (i) }}{=} \rho_{1,2} B^{-1} \rho_{2,2} B \rho_{2,2}^{-1} B \rho_{1,2}^{-1} B^{-1} \rho_{2,1} \stackrel{(v)}{=} \rho_{2,1}$. Finally, for $j=2$ and $k=1$, we have:

$$
\rho_{1,1} B^{-1} \rho_{2,1} \rho_{2,2} \rho_{2,1}^{-1} B \rho_{1,1}^{-1} \stackrel{\text { (i⿱亠䒑) }}{=} \rho_{1,1} B^{-1} \rho_{2,2} \rho_{1,1}^{-1} \stackrel{\text { (iv })}{=} \rho_{2,2} \text {. }
$$

By [6, Theorem 1], the projection $p_{1}: F_{2}\left(\mathbb{T}^{2}\right) \rightarrow \mathbb{T}^{2}$ onto the first coordinate is a locally-trivial fibre space whose fibre may be identified with $\mathbb{T}^{2}-\{*\}$. This gives rise to the following short exact sequence:

$$
1 \longrightarrow \pi_{1}\left(\mathbb{T}^{2}-\{*\}\right) \longrightarrow P_{2}\left(\mathbb{T}^{2}\right) \stackrel{\left(p_{1}\right)_{\#}}{\longrightarrow} \pi_{1}\left(\mathbb{T}^{2}\right) \longrightarrow 1
$$

Remark 13. By [5, Figure 4.1], $\pi_{1}\left(\mathbb{T}^{2}-\{*\}\right)$ is a free group generated by $\rho_{2,1}$ and $\rho_{2,2}$, which we denote by $F\left(\rho_{2,1}, \rho_{2,2}\right)$. The elements $\left(p_{1}\right)_{\#}\left(\rho_{1,1}\right)$ and $\left(p_{1}\right)_{\#}\left(\rho_{1,2}\right)$ generate the free Abelian group $\pi_{1}\left(\mathbb{T}^{2}\right)$ that we shall identify with $\mathbb{Z} \oplus \mathbb{Z}$ under the correspondence of $\left(p_{1}\right)_{\#}\left(\rho_{1,1}\right)\left(\operatorname{resp} .\left(p_{1}\right)_{\#}\left(\rho_{1,2}\right)\right)$ with $(1,0)$ (resp. $(0,1))$.

By Lemma 12(vi), the map $\varphi: \pi_{1}\left(\mathbb{T}^{2}\right) \rightarrow P_{2}\left(\mathbb{T}^{2}\right)$ defined on the generators of $\pi_{1}\left(\mathbb{T}^{2}\right)$ by $\varphi(1,0)=$ $\rho_{1,1} B^{-1} \rho_{2,1}$ and $\varphi(0,1)=\rho_{1,2} B^{-1} \rho_{2,2}$ is a homomorphism that may be seen to be a section for $\left(p_{1}\right)_{\#}$ using Remark 13, By the short exact sequence (10), we conclude that:

$$
P_{2}\left(\mathbb{T}^{2}\right) \cong F\left(\rho_{2,1}, \rho_{2,2}\right) \oplus \mathbb{Z}\left[\rho_{1,1} B^{-1} \rho_{2,1}\right] \oplus \mathbb{Z}\left[\rho_{1,2} B^{-1} \rho_{2,2}\right] .
$$

In particular, the centre of $P_{2}\left(\mathbb{T}^{2}\right)$ is generated by $\rho_{1,1} B^{-1} \rho_{2,1}$ and $\rho_{1,2} B^{-1} \rho_{2,2}$. Let $\sigma$ be the standard generator of $B_{2}\left(\mathbb{T}^{2}\right)$ that swaps the two base points, so $B=\sigma^{2}$. By [8, Section 5], the automorphism $l_{\sigma}: P_{2}\left(\mathbb{T}^{2}\right) \rightarrow P_{2}\left(\mathbb{T}^{2}\right)$ given by conjugation by $\sigma$ satisfies $l_{\sigma}\left(\rho_{1, k}\right)=\rho_{2, k}$ and $l_{\sigma}\left(\rho_{2, k}\right)=B \rho_{1, k} B^{-1}$ for all $k \in\{1,2\}$. Using the decomposition given in (11), for all $k \in\{1,2\}$, we have $l_{\sigma}\left(\rho_{2, k}\right)=$ $B \rho_{2, k}^{-1}\left(\rho_{1, k} B^{-1} \rho_{2, k}\right)$ and $l_{\sigma}\left(\rho_{1, k} B^{-1} \rho_{2, k}\right)=\rho_{1, k} B^{-1} \rho_{2, k}$. By (11) and the fact that $B_{2}\left(\mathbb{T}^{2}\right)$ is generated by $\left\{\sigma, \rho_{1,1}, \rho_{1,2}, \rho_{2,1}, \rho_{2,2}\right\}$, this implies that the centre of $B_{2}\left(\mathbb{T}^{2}\right)$ is generated by $\rho_{1,1} B^{-1} \rho_{2,1}$ and $\rho_{1,2} B^{-1} \rho_{2,2}$. Writing $x=\rho_{2,1}$ and $y=\rho_{2,2}$ and identifying $\mathbb{Z}\left[\rho_{1,1} B^{-1} \rho_{2,1}\right] \oplus \mathbb{Z}\left[\rho_{1,2} B^{-1} \rho_{2,2}\right]$ with $\mathbb{Z} \oplus \mathbb{Z}$, we may summarise the main algebraic properties of $P_{2}\left(\mathbb{T}^{2}\right)$ as follows.

Theorem 14. Up to isomorphism, $P_{2}\left(\mathbb{T}^{2}\right)$ may be written in the form $F(x, y) \oplus \mathbb{Z} \oplus \mathbb{Z}$, and relative to this identification:

(a) the homomorphism $\left(p_{1}\right)_{\#}: P_{2}\left(\mathbb{T}^{2}\right) \rightarrow \pi_{1}\left(\mathbb{T}^{2}\right)$ is projection onto the group $\mathbb{Z} \oplus \mathbb{Z}$, in other words, $\left(p_{1}\right)_{\#}(w, m, n)=(m, n)$ for all $x \in F(x, y)$ and $m, n \in \mathbb{Z}$.

(b) the element $\sigma$ belongs to $B_{2}\left(\mathbb{T}^{2}\right) \backslash P_{2}\left(\mathbb{T}^{2}\right)$ and satisfies $\sigma^{2}=(B, 0,0)$, where $B=\left[x, y^{-1}\right]$.

(c) the images of the generators of $P_{2}\left(\mathbb{T}^{2}\right)$ under the homomorphism $l_{\sigma}: P_{2}\left(\mathbb{T}^{2}\right) \rightarrow P_{2}\left(\mathbb{T}^{2}\right)$ given by conjugating by $\sigma$ are as follows:

$l_{\sigma}(x, 0,0)=\left(B x^{-1}, 1,0\right), l_{\sigma}(y, 0,0)=\left(B y^{-1}, 0,1\right), l_{\sigma}(\mathbb{1}, 1,0)=(\mathbb{1}, 1,0)$ and $l_{\sigma}(\mathbb{1}, 0,1)=(\mathbb{1}, 0,1)$, where $\mathbb{1}$ denotes the trivial element of $F(x, y)$.

The following equation will be used for various computations and arguments in Section 5. Let $|\cdot|_{x},|\cdot|_{y}: F(x, y) \rightarrow \mathbb{Z}$ be the exponent sum homomorphisms that are defined on the generators of $F(x, y)$ by $|x|_{x}=|y|_{y}=1$ and $|x|_{y}=|y|_{x}=0$. By Theorem 14, we have $l_{\sigma}(x, 0,0)=$ $\left(\left(x^{-1} y\right) x^{-1}\left(x^{-1} y\right)^{-1}, 1,0\right)$ and $l_{\sigma}(y, 0,0)=\left(\left(x^{-1} y\right) y^{-1}\left(x^{-1} y\right)^{-1}, 0,1\right)$. From these, if $w=w(x, y) \in$ $F(x, y)$, we obtain the following equality:

$$
l_{\sigma}(w(x, y), 0,0)=\left(x y^{-1} w\left(x^{-1}, y^{-1}\right) y x^{-1},|w|_{x},|w|_{y}\right) .
$$




\section{The braid groups of the Klein bottle}

In this section, we give a presentation of $P_{2}\left(\mathbb{K}^{2}\right)$ in Theorem 16, and we give an explicit description of the semi-direct product structure of this group in Theorem 23. We then determine the action by conjugation of $\sigma$ on the elements of $P_{2}\left(\mathbb{K}^{2}\right)$ in Theorem 24 that will be used in later sections.

If $G$ is a group and $a, b \in G$, we define their anti-commutator by $[a, b]^{\prime}=a b a b^{-1}$. By [1, Theorem A.3], $B_{2}\left(\mathbb{K}^{2}\right)$ has the following presentation.

Theorem 15. The following is a presentation of $B_{2}\left(\mathbb{K}^{2}\right)$ :

generators: $a_{1}, a_{2}, \sigma$.

relations:

(R2) $\sigma^{-1} a_{r} \sigma^{-1} a_{r}=a_{r} \sigma^{-1} a_{r} \sigma$, for all $r \in\{1,2\}$.

(R3) $\sigma^{-1} a_{1} \sigma a_{2}=a_{2} \sigma^{-1} a_{1} \sigma$.

(TR) $a_{1}^{2} a_{2}^{2}=\sigma^{2}$.

In order to exhibit a presentation of $P_{2}\left(\mathbb{K}^{2}\right)$, we apply the Reidemeister-Schreier rewriting process that is described in detail in [12, Chapter 2, Theorem 2.8] and briefly in [14, Appendix I, Theorem 6.3]. We use the notation of [14].

Theorem 16. The following is a presentation of $P_{2}\left(\mathbb{K}^{2}\right)$ :

generators: $a_{1}, a_{2}, b_{1}, b_{2}, B$.

relations:

(i) $b_{r} a_{r}=B a_{r} B^{-1} b_{r} B$, for all $r \in\{1,2\}$.

(ii) $\left[a_{r}, b_{r}\right]=a_{r} B a_{r}^{-1}$, for all $r \in\{1,2\}$.

(iii) $b_{1} B a_{2} B^{-1}=B a_{2} B^{-1} b_{1}$.

(iv) $\left[a_{1}, b_{2}\right]=1$.

(v) $a_{1}^{2} a_{2}^{2}=b_{1}^{2} b_{2}^{2}=B$.

The inclusion $\iota: P_{2}\left(\mathbb{K}^{2}\right) \rightarrow B_{2}\left(\mathbb{K}^{2}\right)$ is defined on the generators of $P_{2}\left(\mathbb{K}^{2}\right)$ by $\iota\left(a_{r}\right)=a_{r}, \iota\left(b_{r}\right)=$ $\sigma a_{r} \sigma^{-1}, r \in\{1,2\}$, and $\iota(B)=\sigma^{2}$.

Proof. First note that $\{\mathbb{1}, \sigma\}$ is a Schreier system of $P_{2}\left(\mathbb{K}^{2}\right)$. Let us compute the generators of $P_{2}\left(\mathbb{K}^{2}\right)$. For $r \in\{1,2\}$, we have:
$\varrho\left(\mathbb{1}, a_{r}\right)=\mathbb{1} a_{r} \overline{\mathbb{1}}^{-1}=a_{r}$.
$\varrho(\mathbb{1}, \sigma)=\mathbb{1} \sigma \overline{\mathbb{1}}^{-1}=\mathbb{1}$.
$\varrho\left(\sigma, a_{r}\right)=\sigma a_{r}{\overline{\sigma a_{r}}}^{-1}=\sigma a_{r} \sigma^{-1}=b_{r}$.
$\varrho(\sigma, \sigma)=\sigma \sigma \overline{\sigma \sigma}^{-1}=\sigma^{2}=B$.

To determine the relations, we apply the Reidemeister-Schreier rewriting process. We leave the straightforward calculations to the reader.

Recall that the projection $p_{1}: F_{2}\left(\mathbb{K}^{2}\right) \rightarrow \mathbb{K}^{2}$ onto the first coordinate is a locally-trivial fibre space whose fibre may be identified with $\mathbb{K}^{2}-\{*\}[6$, Theorem 1], from which we obtain the following short exact sequence:

$$
1 \longrightarrow \pi_{1}\left(\mathbb{K}^{2}-\{*\}\right) \longrightarrow P_{2}\left(\mathbb{K}^{2}\right) \stackrel{\left(p_{1}\right)_{\#}}{\longrightarrow} \pi_{1}\left(\mathbb{K}^{2}\right) \longrightarrow 1
$$

Using [1, Figure 10] and the presentation of $P_{2}\left(\mathbb{K}^{2}\right)$ given by Theorem [16, we conclude that $\pi_{1}\left(\mathbb{K}^{2}-\right.$ $\{*\})$ is the free group generated by $\left\{b_{1}, b_{2}\right\}$, which we denote by $F\left(b_{1}, b_{2}\right)$. For $k \in\{1,2\}$, let $\widetilde{a}_{k}=$ 
$\left(p_{1}\right)_{\#}\left(a_{k}\right)$. By Theorem 16 and the short exact sequence (13), we have $\pi_{1}\left(\mathbb{K}^{2}\right)=\left\langle\widetilde{a}_{1}, \widetilde{a}_{2} \mid \widetilde{a}_{1}^{2} \widetilde{a}_{2}^{2}=\mathbb{1}\right\rangle$. Let $u_{1}=\widetilde{a}_{1} \widetilde{a}_{2}$ and $v_{1}=\widetilde{a}_{2}^{-1}$. Then $\widetilde{a}_{1}=u_{1} v_{1}$ and $\widetilde{a}_{2}=v_{1}^{-1}$. This choice of elements yields another classical presentation of the fundamental group of the Klein bottle of the form $\pi_{1}\left(\mathbb{K}^{2}\right)=$ $\left\langle u_{1}, v_{1} \mid\left[u_{1}, v_{1}\right]^{\prime}=\mathbb{1}\right\rangle=\mathbb{Z}\left[u_{1}\right] \rtimes \mathbb{Z}\left[v_{1}\right]$.

Remark 17. As a set, the group $\mathbb{Z} \rtimes \mathbb{Z}$ is the Cartesian product of $\mathbb{Z}$ with itself. The group operation is given by $\left(r_{1}, s_{1}\right)\left(r_{2}, s_{2}\right)=\left(r_{1}+(-1)^{s_{1}} r_{2}, s_{1}+s_{2}\right)$, the identity element is $(0,0)$, the inverse of an element $(r, s)$ is $\left((-1)^{s+1} r,-s\right)$, and there is a canonical isomorphism between $\mathbb{Z}\left[u_{1}\right] \rtimes \mathbb{Z}\left[v_{1}\right]$ and $\mathbb{Z} \rtimes \mathbb{Z}$. In the rest of this paper, we identify $\pi_{1}\left(\mathbb{K}^{2}\right)$ with $\mathbb{Z} \rtimes \mathbb{Z}$ in this way.

Since $\mathbb{K}^{2}$ admits a non-vanishing vector field, the projection $p_{1}$ admits a geometric section [6], and this implies that the short exact sequence (13) splits. In the following proposition, we give an explicit algebraic section.

Proposition 18. The correspondences $\varphi(1,0)=b_{1} a_{1} b_{2} a_{2}$ and $\varphi(0,1)=a_{2}^{-1} b_{2}^{-1}$ define a homomorphism $\varphi: \pi_{1}\left(\mathbb{K}^{2}\right) \rightarrow P_{2}\left(\mathbb{K}^{2}\right)$ that is a section for the homomorphism $\left(p_{1}\right)_{\#}: P_{2}\left(\mathbb{K}^{2}\right) \rightarrow \pi_{1}\left(\mathbb{K}^{2}\right)$.

Proof. We have that:

$$
\begin{aligned}
{[\varphi(1,0), \varphi(0,1)]^{\prime} } & =b_{1} a_{1} b_{1} a_{1} b_{2} a_{2} b_{2} a_{2}=\left[b_{1}, a_{1}\right] a_{1} b_{1}^{2} a_{1} b_{2} a_{2}\left[b_{2}, a_{2}\right] a_{2} b_{2} \\
& \stackrel{(\mathrm{ii})}{=} a_{1} B^{-1} b_{1}^{2} a_{1} b_{2} a_{2}^{2} B^{-1} b_{2} \stackrel{(\mathrm{v})}{=} a_{1} b_{2}^{-2} a_{1} b_{2} a_{1}^{-2} b_{2} \stackrel{\text { (iv) }}{=} \mathbb{1} .
\end{aligned}
$$

where we make use of the relations given in Theorem 16. So by Remark 17, $\varphi$ is a well-defined homomorphism. Using the relations $\widetilde{a}_{1}=u_{1} v_{1}$ and $\widetilde{a}_{2}=v_{1}^{-1}$, it follows that $\varphi$ is a section for $\left(p_{1}\right)_{\# \cdot} \cdot$

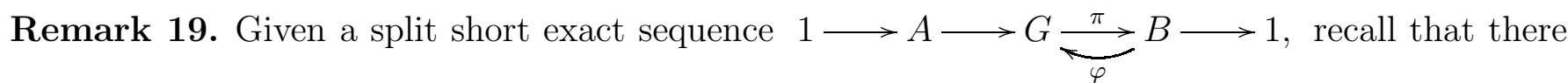
exists a homomorphism $\theta: B \rightarrow \operatorname{Aut}(A)$, defined by $\theta(b)(a)=\varphi(b) a \varphi(b)^{-1}$, and isomorphisms $A \rtimes_{\theta} B \underset{\gamma}{\stackrel{\lambda}{\rightleftarrows}} G$, defined by $\lambda(a, b)=a \varphi(b)$ and $\gamma(g)=\left(g \varphi(\pi(g))^{-1}, \pi(g)\right)$.

Proposition 18 and Remark 19 imply that $P_{2}\left(\mathbb{K}^{2}\right) \cong F\left(b_{1}, b_{2}\right) \rtimes_{\theta}(\mathbb{Z} \rtimes \mathbb{Z})$. In the following result, we describe the action by conjugation of $a_{r}$ on the elements $b_{k}$ and $B$ for all $r, k \in\{1,2\}$.

Lemma 20. In $P_{2}\left(\mathbb{K}^{2}\right)$, the following relations hold:
(1) $a_{1} b_{1} a_{1}^{-1}=b_{1}^{-1} b_{2}^{-2}$.
(4) $a_{2} b_{1} a_{2}^{-1}=b_{2}^{-1} B^{-1} b_{2}^{-1} b_{1} b_{2} B b_{2}$.
(2) $a_{1} b_{2} a_{1}^{-1}=b_{2}$.
(5) $a_{2} b_{2} a_{2}^{-1}=b_{2}^{-1} B^{-1} b_{2}^{2}$.
(3) $a_{1} B a_{1}^{-1}=b_{1}^{-1} B^{-1} b_{1}$.
(6) $a_{2} B a_{2}^{-1}=b_{2}^{-1} B^{-1} b_{2}$.

Proof. First, we have:

$$
\left(a_{1} b_{1} a_{1}\right)^{2} \stackrel{(\mathrm{v})}{=} a_{1} B b_{2}^{-2} a_{1}^{-1} \stackrel{(\mathrm{iv})}{=} a_{1} B a_{1}^{-1} b_{2}^{-2} \stackrel{\text { (ii) }}{=}\left(a_{1} b_{1} a_{1}^{-1}\right) b_{1}^{-1} b_{2}^{-2} .
$$

This equality implies relation (1). Relation (2) follows directly from (iv). Further:

$$
a_{1} B a_{1}^{-1} \stackrel{(\mathrm{v})}{=} a_{1} b_{1}^{2} b_{2}^{2} a_{1}^{-1} \stackrel{(\mathrm{iv})}{=}\left(a_{1} b_{1} a_{1}^{-1}\right)^{2} b_{2}^{2} \stackrel{(1)}{=} b_{1}^{-1} b_{2}^{-2} b_{1}^{-1} \stackrel{(\mathrm{v})}{=} b_{1}^{-1} B^{-1} b_{1}
$$

which proves relation (3). It remains to prove relations (4), (5) and (6). We have:

$$
\begin{aligned}
b_{1} B a_{2} B^{-1} a_{2}^{-1} & \stackrel{\text { (iii) }}{=} B a_{2} B^{-1} b_{1} a_{2}^{-1}=B\left(a_{2} B^{-1} a_{2}^{-1}\right)\left(a_{2} b_{1} a_{2}^{-1}\right), \text { which implies that } \\
a_{2} b_{1} a_{2}^{-1} & =a_{2} B a_{2}^{-1} B^{-1} a_{2} B^{-1} a_{2}^{-1},
\end{aligned}
$$


and

$$
a_{2} b_{2} a_{2}^{-1}=\left[a_{2}, b_{2}\right] b_{2} \stackrel{(\text { ii) }}{=} a_{2} B a_{2}^{-1} b_{2}
$$

Using (14) and (15), we obtain:

$$
a_{2} B a_{2}^{-1} \stackrel{(\mathrm{v})}{=}\left(a_{2} b_{1} a_{2}\right)^{2}\left(a_{2} b_{2} a_{2}\right)^{2}=a_{2} B a_{2}^{-1} B^{-1} b_{1}^{2} B b_{2} a_{2} B a_{2}^{-1} b_{2} \stackrel{(\mathrm{v})}{=}\left(a_{2} B a_{2}^{-1}\right) b_{2}^{-2} B b_{2}\left(a_{2} B a_{2}^{-1}\right) b_{2},
$$

which implies relation (6). Using relation (6), relations (4) and (5) then follow from (14) and (15) respectively.

To simplify various computations, it will be convenient to carry out the change of basis given by $u=b_{1} b_{2}, v=b_{2}^{-1}$, so $b_{1}=u v$ and $b_{2}=v^{-1}$. Note that in the new basis, $B=[u, v]^{\prime}$. In order to determine the automorphism $\theta(m, n): F(u, v) \rightarrow F(u, v)$ for each $(m, n) \in \mathbb{Z} \rtimes \mathbb{Z}$, we require the following two lemmas.

Lemma 21. For all $m \in \mathbb{Z}$, we have:

$$
\theta(m, 0):\left\{\begin{array}{l}
u \longmapsto B^{m} u B^{-m} \\
v \longmapsto B^{m} v u^{-2 m} B^{-m} \\
B \longmapsto B .
\end{array}\right.
$$

Proof. The result is clear if $m=0$. If $m=1$ then Proposition 18 and Lemma 20 imply that:

$$
\begin{aligned}
\theta(1,0)(u) & =\varphi(1,0) b_{1} b_{2} \varphi(1,0)^{-1}=b_{1} a_{1} b_{2} a_{2} b_{1} b_{2} a_{2}^{-1} b_{2}^{-1} a_{1}^{-1} b_{1}^{-1}=b_{1} a_{1} B^{-1} b_{2}^{-1} b_{1} a_{1}^{-1} b_{1}^{-1} \\
& =B b_{1} b_{2}^{-1} b_{1}^{-1} b_{2}^{-2} b_{1}^{-1}=B u v u^{-1} v^{-1} u^{-1}=B u B^{-1} \\
\theta(1,0)(v) & =\varphi(1,0) b_{2}^{-1} \varphi(1,0)^{-1}=b_{1} a_{1} b_{2} a_{2} b_{2}^{-1} a_{2}^{-1} b_{2}^{-1} a_{1}^{-1} b_{1}^{-1}=b_{1} a_{1} b_{2}^{-1} B a_{1}^{-1} b_{1}^{-1} \\
& =b_{1} b_{2}^{-1} b_{1}^{-1} B^{-1}=u v u^{-1} B^{-1}=B v u^{-2} B^{-1}, \text { and } \\
\theta(1,0)(B) & =\varphi(1,0) B \varphi(1,0)^{-1}=b_{1} a_{1} b_{2} a_{2} B a_{2}^{-1} b_{2}^{-1} a_{1}^{-1} b_{1}^{-1}=b_{1} a_{1} B^{-1} a_{1}^{-1} b_{1}^{-1}=B .
\end{aligned}
$$

Suppose that the result holds for all integers belonging to $\{1, \ldots, m\}$, and let us show by induction that it holds for $m+1$. We have:

$$
\begin{aligned}
\theta(m+1,0)(u) & =\theta(m, 0)(\theta(1,0)(u))=\theta(m, 0)\left(B u B^{-1}\right)=B^{m+1} u B^{-(m+1)}, \\
\theta(m+1,0)(v) & =\theta(m, 0)(\theta(1,0)(v))=\theta(m, 0)\left(B v u^{-2} B^{-1}\right)=B^{m+1} v u^{-2(m+1)} B^{-(m+1)}, \text { and } \\
\theta(m+1,0)(B) & =\theta(m, 0)(\theta(1,0)(B))=\theta(m, 0)(B)=B,
\end{aligned}
$$

as required, and so the result holds for all $m \geq 0$. Now suppose that $m<0$. Then $-m>0$, and so:

$$
B=\theta(0,0)(B)=\theta(m, 0)(\theta(-m, 0)(B))=\theta(m, 0)(B) .
$$

We also have:

$$
u=\theta(m, 0)(\theta(-m, 0)(u))=\theta(m, 0)\left(B^{-m} u B^{m}\right)=B^{-m} \theta(m, 0)(u) B^{m},
$$

thus $\theta(m, 0)=B^{m} u B^{-m}$, and:

$$
v=\theta(m, 0)(\theta(-m, 0)(v))=\theta(m, 0)\left(B^{-m} v u^{2 m} B^{m}\right)=B^{-m} \theta(m, 0)(v) B^{m} u^{2 m},
$$

hence $\theta(m, 0)(v)=B^{m} v u^{-2 m} B^{-m}$ as required.

Lemma 22. For all $n \in \mathbb{Z}$, we have: 


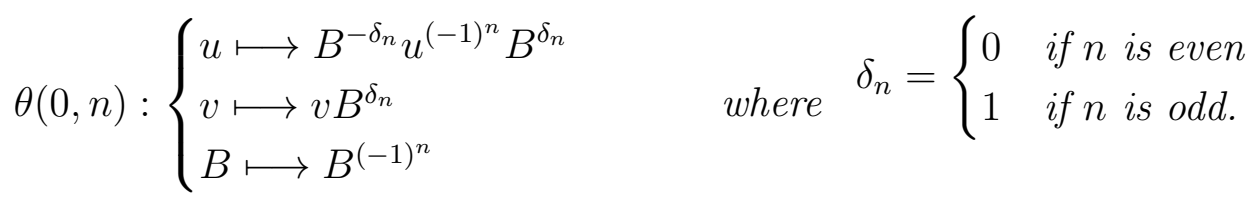

Proof. By relation (v) of Theorem [16, we have $\varphi(0,1)=B^{-1} a_{1}^{2} a_{2} b_{2}^{-1}$. Using this equality, the idea of the proof is similar to that of Lemma 21, and we leave the calculations to the reader.

We are now able to describe the homomorphism $\theta$.

Theorem 23. The homomorphism $\theta: \mathbb{Z} \rtimes \mathbb{Z} \rightarrow A u t(F(u, v))$ is defined by:

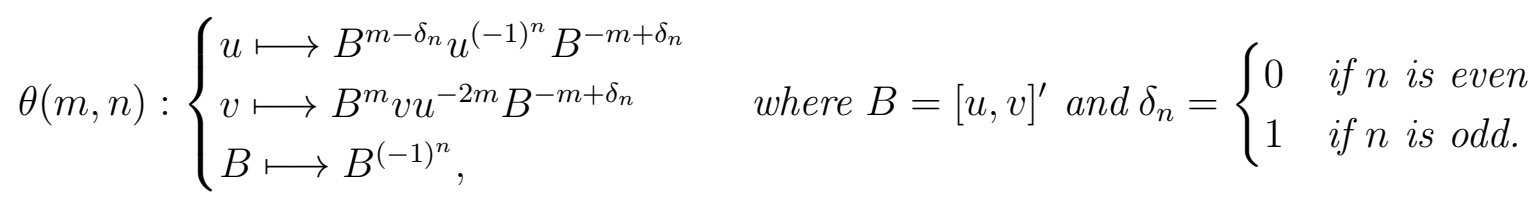

With the notation of Remark 19 , the isomorphisms $F(u, v) \rtimes_{\theta}(\mathbb{Z} \rtimes \mathbb{Z}) \underset{\gamma}{\stackrel{\lambda}{\rightleftarrows}} P_{2}\left(\mathbb{K}^{2}\right)$ are given by:

$$
\lambda:\left\{\begin{array}{l}
(u ; 0,0) \longmapsto b_{1} b_{2} \\
(v ; 0,0) \longmapsto b_{2}^{-1} \\
(B ; 0,0) \longmapsto B \\
(\mathbb{1} ; 1,0) \longmapsto b_{1} a_{1} b_{2} a_{2} \\
(\mathbb{1} ; 0,1) \longmapsto b_{2} a_{2}
\end{array} \quad \gamma:\left\{\begin{array}{l}
a_{1} \longmapsto\left(v^{-1} u^{-1} ; 1,1\right) \\
a_{2} \longmapsto(v ; 0,-1) \\
b_{1} \longmapsto(u v ; 0,0) \\
b_{2} \longmapsto(v-1 ; 0,0) \\
B \longmapsto(B ; 0,0) .
\end{array}\right.\right.
$$

Up these isomorphisms and the identification given by Remark 17, the homomorphism $\left(p_{1}\right)_{\#}$ : $P_{2}\left(\mathbb{K}^{2}\right) \rightarrow \pi_{1}\left(\mathbb{K}^{2}\right)$ is the projection onto the second coordinate, in other words, $\left(p_{1}\right)_{\#}(w ; m, n)=$ $(m, n)$.

Proof. For all $(m, n) \in \mathbb{Z} \rtimes \mathbb{Z}$, we have $\theta(m, n)=\theta(m, 0) \circ \theta(0, n)$. The first part of the statement follows by combining Lemmas 21 and 22 . Let us prove the second part. First, $\lambda(u ; 0,0)=u=b_{1} b_{2}$, $\lambda(v ; 0,0)=v=b_{2}^{-1}, \lambda(B ; 0,0)=B, \lambda(\mathbb{1} ; 1,0)=\mathbb{1} \varphi(1,0)=b_{1} a_{1} b_{2} a_{2}$ and $\lambda(\mathbb{1} ; 0,1)=\mathbb{1} \varphi(0,1)=$ $a_{2}^{-1} b_{2}^{-1}$. On the other hand:

$$
\begin{aligned}
& \gamma\left(a_{1}\right)=\left(a_{1} \varphi\left(\widetilde{a}_{1}\right)^{-1} ; \widetilde{a}_{1}\right)=\left(a_{1}(\varphi(1,0) \varphi(0,1))^{-1} ; 1,1\right)=\left(b_{1}^{-1} ; 1,1\right)=\left(v^{-1} u^{-1} ; 1,1\right) \\
& \gamma\left(a_{2}\right)=\left(a_{2} \varphi\left(\widetilde{a}_{2}\right)^{-1} ; \widetilde{a}_{2}\right)=\left(a_{2} \varphi(0,1) ; 0,-1\right)=\left(b_{2}^{-1} ; 0,-1\right)=(v ; 0,-1),
\end{aligned}
$$

$\gamma\left(b_{1}\right)=(u v ; 0,0)$ and $\gamma\left(b_{2}\right)=\left(v^{-1} ; 0,0\right)$. From the isomorphism $\lambda$ and Remark 17, we see that $\left(p_{1}\right)_{\#}(u ; 0,0)=\left(p_{1}\right)_{\#}(v ; 0,0)=(0,0),\left(p_{1}\right)_{\#}(\mathbb{1} ; 1,0)=(1,0)$ and $\left(p_{1}\right)_{\#}(\mathbb{1} ; 0,1)=(0,1)$ as required.

Let $c_{\sigma}: P_{2}\left(\mathbb{K}^{2}\right) \rightarrow P_{2}\left(\mathbb{K}^{2}\right)$ be the automorphism of $P_{2}(T)$ given by conjugation by $\sigma$. By Theorem 16, we have $c_{\sigma}(B)=B, c_{\sigma}\left(a_{i}\right)=b_{i}$ and $c_{\sigma}\left(b_{j}\right)=B a_{j} B^{-1}$ for all $i, j \in\{1,2\}$. Let $l_{\sigma}: F(u, v) \rtimes_{\theta}(\mathbb{Z} \rtimes \mathbb{Z}) \rightarrow F(u, v) \rtimes_{\theta}(\mathbb{Z} \rtimes \mathbb{Z})$ be defined by the composition $l_{\sigma}=\gamma \circ c_{\sigma} \circ \lambda$. To end this section, we give an explicit description of $l_{\sigma}$ on a set of generators.

Theorem 24. For all $r, s, m, n \in \mathbb{Z}$, we have:

(1) $l_{\sigma}\left(u^{r} ; 0,0\right)=\left(\left(B u^{-1}\right)^{-r} B^{-r} ; r, 0\right)$.

(4) $l_{\sigma}(\mathbb{1} ; m, 0)=(\mathbb{1} ; m, 0)$.

(2) $l_{\sigma}\left(v^{s} ; 0,0\right)=\left((u v)^{-s}(u B)^{\delta_{s}} ; 0, s\right)$.

(5) $l_{\sigma}(\mathbb{1} ; 0, n)=\left(B^{\delta_{n}} ; 0, n\right)$.

(3) $l_{\sigma}(B ; 0,0)=(B ; 0,0)$. 
Proof. Equation (3) follows easily from Theorem 23. Let us prove equation (1). For $r=0$, the result is clear. If $r=1$, we have:

$$
\begin{aligned}
l_{\sigma}(u ; 0,0) & =\left(\gamma \circ c_{\sigma}\right)\left(b_{1} b_{2}\right)=\gamma\left(B a_{1} a_{2} B^{-1}\right)=(B ; 0,0)\left(v^{-1} u^{-1} ; 1,1\right)(v ; 0,-1)\left(B^{-1}, 0,0\right) \\
& =\left(B v^{-1} u^{-1} \theta(1,1)(v) \theta(1,0)(B)^{-1} ; 1,0\right)=\left(B v^{-1} u^{-1} B v u^{-2} B^{-1} ; 1,0\right)=\left(B u^{-1} B^{-1} ; 1,0\right) .
\end{aligned}
$$

Now suppose that the result holds for all integers belonging to $\{1, \ldots, r\}$, and let us show by induction that it is true for $r+1$. We have:

$$
\begin{aligned}
l_{\sigma}\left(u^{r+1} ; 0,0\right) & =l_{\sigma}(u ; 0,0) l_{\sigma}\left(u^{r} ; 0,0\right)=\left(B u^{-1} B^{-1} ; 1,0\right)\left(\left(B u^{-1}\right)^{r} B^{-r} ; r, 0\right) \\
& =\left(B u^{-1} B^{-1}\left(\theta(1,0)(B) \theta(1,0)(u)^{-1}\right)^{r} \theta(1,0)(B)^{-r} ; r+1,0\right) \\
& =\left(B u^{-1} B^{-1}\left(B\left(B u B^{-1}\right)^{-1}\right)^{r} B^{-r} ; r+1,0\right)=\left(\left(B u^{-1}\right)^{r+1} B^{-(r+1)} ; r, 0\right) .
\end{aligned}
$$

and hence equation (1) holds for all $r \geq 0$. Now assume that $r<0$. Then $-r>0$, and since $l_{\sigma}(u ; 0,0)=l_{\sigma}\left(u^{-r} ; 0,0\right)^{-1}$, it follows that:

$$
\begin{aligned}
l_{\sigma}\left(u^{r} ; 0,0\right) & =l_{\sigma}\left(u^{-r} ; 0,0\right)^{-1}=\left(\left(B u^{-1}\right)^{-r} B^{r} ;-r, 0\right)^{-1}=\left(\left(\theta(r, 0)\left(\left(B u^{-1}\right)^{-r} B^{r}\right)\right)^{-1} ; r, 0\right) \\
& =\left(\left(\left(\theta(r, 0)(B) \theta(r, 0)(u)^{-1}\right)^{-r} \theta(r, 0)(B)^{r}\right)^{-1} ; r, 0\right) \\
& =\left(\left(\left(B\left(B^{r} u B^{-r}\right)^{-1}\right)^{-r} B^{r}\right)^{-1} ; r, 0\right)=\left(\left(B u^{-1}\right)^{r} B^{-r} ; r, 0\right) .
\end{aligned}
$$

This proves equation (1). Let us show that equation (2) holds. If $s=0$, the result is immediate. For $s=1,2$, we have:

$$
\begin{aligned}
l_{\sigma}(v ; 0,0) & =\left(\gamma \circ c_{\sigma}\right)\left(b_{2}^{-1}\right)=\gamma\left(B a_{2}^{-1} B^{-1}\right)=(B ; 0,0)(v ; 0,-1)^{-1}(B ; 0,0)^{-1} \\
& =(B ; 0,0)\left(\theta(0,1)(v)^{-1} ; 0,1\right)\left(B^{-1} ; 0,0\right)=\left(B(v B)^{-1} \theta(0,1)(B)^{-1} ; 0,1\right) \\
& =\left(v^{-1} B ; 0,1\right)=\left((u v)^{-1}(u B)^{\delta_{1}} ; 0,1\right), \text { and } \\
l_{\sigma}\left(v^{2} ; 0,0\right) & =l_{\sigma}(v ; 0,0) l_{\sigma}(v ; 0,0)=\left(v^{-1} B ; 0,1\right)\left(v^{-1} B ; 0,1\right)=\left(v^{-1} B \theta(0,1)(v)^{-1} \theta(0,1)(B) ; 0,2\right) \\
& =\left(\left(v^{-1} B(v B)^{-1} B^{-1} ; 0,2\right)=\left(v^{-2} B^{-1} ; 0,2\right)=\left((u v)^{-2}(u B)^{\delta_{2}} ; 0,2\right),\right.
\end{aligned}
$$

and so equation (2) holds in these two cases. Now suppose that $s$ is even. Then $s=2 k$ for some $k \in \mathbb{Z}$. We wish to show that $l_{\sigma}\left(v^{2 k} ; 0,0\right)=\left((u v)^{-2 k} ; 0,2 k\right)$. If $k=1$, the result holds by (17). Suppose that $k \geq 1$ and that the result is true for all integers belonging to $\{1, \ldots, k\}$. Then:

$$
\begin{aligned}
l_{\sigma}\left(v^{2(k+1)} ; 0,0\right) & =l_{\sigma}\left(v^{2 k} ; 0,0\right) l_{\sigma}\left(v^{2} ; 0,0\right)=\left((u v)^{-2 k} ; 0,2 k\right)\left((u v)^{-2} ; 0,2\right) \\
& =\left((u v)^{-2 k}(\theta(0,2 k)(u) \theta(0,2 k)(v))^{-2} ; 0,2 k+2\right) \\
& =\left((u v)^{-2 k}(u v)^{-2} ; 0,2(k+1)\right)=\left((u v)^{-2(k+1)} ; 0,2(k+1)\right) .
\end{aligned}
$$

and so by induction, equation (2) holds for all $k \geq 1$. Now suppose that $k<0$. Then $-k>0$, and since $l_{\sigma}\left(v^{2 k}, 0,0\right)=l_{\sigma}\left(v^{-2 k} ; 0,0\right)^{-1}$, we see that:

$$
\begin{aligned}
l_{\sigma}\left(v^{2 k} ; 0,0\right) & =l_{\sigma}\left(v^{-2 k} ; 0,0\right)^{-1}=\left((u v)^{2 k} ; 0,-2 k\right)^{-1}=\left(\left(\theta(0,2 k)\left((u v)^{2 k}\right)\right)^{-1} ; 0,2 k\right) \\
& =\left(\left((\theta(0,2 k)(u) \theta(0,2 k)(v))^{2 k}\right)^{-1} ; 0,2 k\right)=\left((u v)^{-2 k} ; 0,2 k\right),
\end{aligned}
$$

and hence equation (2) holds for all $s$ even. Now assume that $s$ is odd, and let $k \in \mathbb{Z}$ be such that $s=2 k+1$. Using (16) and the result for $s$ even, we obtain:

$$
\begin{aligned}
l_{\sigma}\left(v^{2 k+1} ; 0,0\right) & =l_{\sigma}\left(v^{2 k} ; 0,0\right) l_{\sigma}(v ; 0,0)=\left((u v)^{-2 k} ; 0,2 k\right)\left(v^{-1} B ; 0,1\right) \\
& =\left((u v)^{-2 k} \theta(0,2 k)(v)^{-1} \theta(0,2 k)(B) ; 0,2 k+1\right) \\
& =\left((u v)^{-2 k} v^{-1} B ; 0, s\right)=\left((u v)^{-s}(u B)^{\delta_{s}}, 0, s\right) .
\end{aligned}
$$


This completes the proof of equation (2). To obtain equation (4), it suffices to prove the result for $m=1$. We have:

$$
\begin{aligned}
l_{\sigma}(\mathbb{1} ; 1,0) & =\left(\gamma \circ c_{\sigma}\right)\left(b_{1} a_{1} b_{2} a_{2}\right)=\gamma\left(B a_{1} B^{-1} b_{1} B a_{2} B^{-1} b_{2}\right) \\
& =(B ; 0,0)\left(v^{-1} u^{-1} ; 1,1\right)(B ; 0,0)^{-1}(u v ; 0,0)(B ; 0,0)(v ; 0,-1)(B ; 0,0)^{-1}\left(v^{-1} ; 0,0\right) \\
& =\left(B v^{-1} u^{-1} \theta(1,1)(B)^{-1} \theta(1,1)(u) \theta(1,1)(v) \theta(1,1)(B) \theta(1,1)(v) \theta(1,0)(B)^{-1} \theta(1,0)(v)^{-1} ; 1,0\right) \\
& =\left(B v^{-1} u^{-1} B u^{-1} B v u^{-2} B^{-1} B v u^{-2} B^{-1}\left(B v u^{-2} B^{-1}\right)^{-1} ; 1,0\right)=(\mathbb{1} ; 1,0),
\end{aligned}
$$

as required. Finally, to prove equation (5), if $n \in \mathbb{Z}$, let $k \in \mathbb{Z}$ be such that $n=2 k+\delta_{n}$. Then:

$$
\begin{aligned}
l_{\sigma}(\mathbb{1} ; 0, n) & =l_{\sigma}(\mathbb{1} ; 0,1)^{n}=(B ; 0,1)^{n}=(B ; 0,1)^{2 k}(B ; 0,1)^{\delta_{n}} \\
& =((B ; 0,1)(B ; 0,1))^{k}\left(B^{\delta_{n}} ; 0, \delta_{n}\right)=(B \theta(0,1)(B) ; 0,2)^{k}\left(B^{\delta_{n}}, 0, \delta_{n}\right) \\
& =(\mathbb{1} ; 0,2 k)\left(B^{\delta_{n}} ; 0, \delta_{n}\right)=\left(\theta(0,2 n)(B)^{\delta_{n}} ; 0,2 k+\delta_{n}\right)=\left(B^{\delta_{n}} ; 0, n\right),
\end{aligned}
$$

and thus equation (5) holds.

\section{Proof of Theorems 1 and 2}

The purpose of this section is to prove Theorems 1 and 2 . We identify $\pi_{1}\left(\mathbb{T}^{2}\right)$ with $\mathbb{Z} \oplus \mathbb{Z}$ and $\pi_{1}\left(\mathbb{K}^{2}\right)$ with $\mathbb{Z} \rtimes \mathbb{Z}$ as in Sections 3 and 4 respectively. Consider the following short exact sequences:

$$
\begin{aligned}
1 \longrightarrow \pi_{1}\left(\mathbb{T}^{2}\right) \stackrel{i_{1}}{\longrightarrow} \pi_{1}\left(\mathbb{T}^{2}\right) \stackrel{\theta_{1}}{\longrightarrow} \mathbb{Z}_{2} \longrightarrow 1, \text { where: } & i_{1}:\left\{\begin{array}{l}
(1,0) \longmapsto(2,0) \\
(0,1) \longmapsto(0,1)
\end{array} \theta_{1}:\left\{\begin{array}{l}
(1,0) \longmapsto \overline{1} \\
(0,1) \longmapsto \overline{0}
\end{array}\right.\right.
\end{aligned}
$$

and

$$
\begin{aligned}
1 \longrightarrow \pi_{1}\left(\mathbb{T}^{2}\right) \stackrel{i_{2}}{\longrightarrow} \pi_{1}\left(\mathbb{K}^{2}\right) \stackrel{\theta_{2}}{\longrightarrow} \mathbb{Z}_{2} \longrightarrow 1, \text { where: } \\
i_{2}:\left\{\begin{array}{l}
(1,0) \longmapsto(1,0) \\
(0,1) \longmapsto(0,2)
\end{array} \theta_{2}:\left\{\begin{array}{l}
(1,0) \longmapsto \overline{0} \\
(0,1) \longmapsto \overline{1} .
\end{array}\right.\right.
\end{aligned}
$$

By covering space theory, there exist double coverings $c_{1}: \mathbb{T}^{2} \rightarrow \mathbb{T}^{2}$ and $c_{2}: \mathbb{T}^{2} \rightarrow \mathbb{K}^{2}$ such that the induced homomorphisms on the fundamental group are $i_{1}$ and $i_{2}$ respectively. If $k \in\{1,2\}$ and $\tau_{k}: \mathbb{T}^{2} \rightarrow \mathbb{T}^{2}$ is the non-identity deck transformation associated with $c_{k}$, then $\tau_{k}$ is a free involution, and the short exact sequence induced by $\tau_{k}$ is (18) if $k=1$ and is (19) if $k=2$. Further, $\tau_{k}$ lifts to a homeomorphism $\widehat{\tau}_{k}: \mathbb{R}^{2} \rightarrow \mathbb{R}^{2}$, where $\widehat{\tau}_{1}(x, y)=\left(x+\frac{1}{2}, y\right)$ and $\widehat{\tau}_{2}(x, y)=\left(x+\frac{1}{2}, 1-y\right)$ for all $(x, y) \in \mathbb{R}^{2}$. As we shall now see, up to the equivalence relation defined just after Proposition 9 , these are the only free involutions of the torus.

Proposition 25. The free involutions $\tau_{1}$ and $\tau_{2}$ are not equivalent. Further, let $\tau: \mathbb{T}^{2} \rightarrow \mathbb{T}^{2}$ be a free involution. If $\tau$ preserves (resp. reverses) orientation, then $\tau$ is equivalent to $\tau_{1}$ (resp. to $\tau_{2}$ ).

Proof. The short exact sequences (18) and (19) are not equivalent (in the sense described at the end of Section 2), since the middle groups are not isomorphic, and so $\tau_{1}$ and $\tau_{2}$ are not equivalent by Proposition 10. Now let $\tau: \mathbb{T}^{2} \rightarrow \mathbb{T}^{2}$ be a free involution. Recall that $\mathbb{T}_{\tau}^{2}$ is the corresponding orbit space, and that the natural projection $p_{\tau}: \mathbb{T}^{2} \rightarrow \mathbb{T}_{\tau}^{2}$ is a double covering. Suppose first that $\tau$ preserves orientation. Then $\mathbb{T}_{\tau}^{2}$ is homeomorphic to the torus, and the short exact sequence induced by $\tau$ is of the following form:

$$
1 \longrightarrow \pi_{1}\left(\mathbb{T}^{2}\right) \stackrel{\left(p_{\tau}\right)_{\#}}{\longrightarrow} \underbrace{\pi_{1}\left(\mathbb{T}_{\tau}^{2}\right)}_{\cong \mathbb{Z} \oplus \mathbb{Z}} \stackrel{\theta_{\tau}}{\longrightarrow} \mathbb{Z}_{2} \longrightarrow 1 .
$$


By [8, Proposition 30], there exists an isomorphism $\psi: \pi_{1}\left(\mathbb{T}^{2}\right) \rightarrow \pi_{1}\left(\mathbb{T}_{\tau}^{2}\right)$ such that $\theta_{\tau} \circ \psi=\theta_{1}$. Comparing diagrams (18) and (20) and using exactness and the 5-Lemma, it follows that $\psi$ induces an isomorphism $\varphi: \pi_{1}\left(\mathbb{T}^{2}\right) \rightarrow \pi_{1}\left(\mathbb{T}^{2}\right)$ such that $\left(p_{\tau}\right)_{\#} \circ \varphi=\psi \circ i_{1}$. So the exact sequences (18) and (20) are equivalent, and we conclude that $\tau$ and $\tau_{1}$ are equivalent by Proposition 10. Now suppose that $\tau$ reverses orientation. Then $\mathbb{T}_{\tau}^{2}$ is homeomorphic to the Klein bottle, and the short exact sequence induced by $\tau$ is of the following form:

$$
1 \longrightarrow \pi_{1}\left(\mathbb{T}^{2}\right) \stackrel{\left(p_{\tau}\right)_{\#}}{\longrightarrow} \underbrace{\pi_{1}\left(\mathbb{T}_{\tau}^{2}\right)}_{\cong \mathbb{Z} \rtimes \mathbb{Z}} \stackrel{\theta_{\tau}}{\longrightarrow} \mathbb{Z}_{2} \longrightarrow 1 .
$$

Using the identification described in Remark 17, we have an isomorphism $\gamma: \mathbb{Z} \rtimes \mathbb{Z} \rightarrow \pi_{1}\left(\mathbb{T}_{\tau}^{2}\right)$. The elements $\gamma(0,1)=v_{1}$ and $\gamma(1,1)=u_{1} v_{1}$ represent loops in $\mathbb{T}_{\tau}^{2}$ that reverse orientation. Since $p_{\tau}: \mathbb{T}^{2} \rightarrow \mathbb{T}_{\tau}^{2}$ is a covering, we have $\theta_{\tau} \circ \gamma(1,0)=\theta_{\tau} \circ \gamma(1,1)=\overline{1}$, so the element $(1,0)=(1,1)(0,1)^{-1}$ satisfies $\theta_{\tau} \circ \gamma(1,0)=\overline{0}$, and thus $\theta_{\tau} \circ \gamma=\theta_{2}$. Let $\psi: \pi_{1}\left(\mathbb{K}^{2}\right) \rightarrow \pi_{1}\left(\mathbb{T}_{\tau}^{2}\right)$ be an isomorphism. Comparing diagrams (19) and (21) and using exactness and the 5-Lemma once more, it follows that $\psi$ induces an isomorphism $\varphi: \pi_{1}\left(\mathbb{T}^{2}\right) \rightarrow \pi_{1}\left(\mathbb{T}^{2}\right)$ such that $\left(p_{\tau}\right)_{\#} \circ \varphi=\psi \circ i_{2}$. So the exact sequences (19) and (21) are equivalent, and we conclude that $\tau$ and $\tau_{2}$ are equivalent by Proposition 10.

Theorem 4 gives rise to the following commutative diagram, where the maps are bijections (we simplify the notation):

$$
\left[\mathbb{T}^{2}, * ; \mathbb{T}^{2}, *\right] \stackrel{\Lambda}{\stackrel{\Gamma o m}{\longrightarrow}(\mathbb{Z} \oplus \mathbb{Z}, \mathbb{Z} \oplus \mathbb{Z}) \stackrel{\Delta}{\longleftarrow}}\left[\mathbb{T}^{2}, \mathbb{T}^{2}\right] .
$$

We shall now solve the Borsuk-Ulam problem for homotopy classes with respect to the involution $\tau_{1}$. We first state and prove the following lemma.

Lemma 26. A free homotopy class $\beta \in\left[\mathbb{T}^{2}, \mathbb{T}^{2}\right]$ does not have the Borsuk-Ulam property with respect to $\tau_{1}$ if and only if there exist pure braids $a, b \in P_{2}\left(\mathbb{T}^{2}\right)$ such that:

(i) $a l_{\sigma}(b)=b a$.

(ii) $\beta_{\#}(1,0)=\left(p_{1}\right)_{\#}\left(a l_{\sigma}(a)\right)$.

(iii) $\beta_{\#}(0,1)=\left(p_{1}\right)_{\#}(b)$.

Proof. Suppose that $\beta$ does not have the Borsuk-Ulam property with respect to $\tau_{1}$. By (22) and Proposition 7, there exists a pointed homotopy class $\alpha$ that does not have the Borsuk-Ulam property and for which $\alpha_{\mathcal{F}}=\Lambda(\alpha)=\beta$. Using Proposition 6 , there exist homomorphisms $\varphi: \pi_{1}\left(\mathbb{T}^{2}\right) \rightarrow P_{2}\left(\mathbb{T}^{2}\right)$ and $\psi: \pi_{1}\left(\mathbb{T}^{2}\right) \rightarrow B_{2}\left(\mathbb{T}^{2}\right)$ that make diagram (9) commute. By (18), $\psi(1,0) \in B_{2}\left(\mathbb{T}^{2}\right) \backslash P_{2}\left(\mathbb{T}^{2}\right)$ and $\psi(0,1) \in P_{2}\left(\mathbb{T}^{2}\right)$. From the short exact sequence (8) and Theorem 14, there exist $a, b \in P_{2}\left(\mathbb{T}^{2}\right)$ such that:

(1) $\psi(1,0)=a \sigma$.

(2) $\psi(0,1)=b$.

Since $\pi_{1}\left(\mathbb{T}^{2}\right)$ is an Abelian group, we have $[\psi(1,0), \psi(0,1)]=a \sigma b \sigma a^{-1} a^{-1} b^{-1}=\mathbb{1}$, which is equivalent to $a l_{\sigma}(b)=b a$, from which we obtain (i). The commutativity of (9) implies that:

(3) $\varphi(1,0)=\psi\left(\left(p_{\tau_{1}}\right)_{\#}(1,0)\right)=\psi(2,0)=(a \sigma)^{2}=a l_{\sigma}(a) \sigma^{2}$, and

(4) $\varphi(0,1)=\psi\left(\left(p_{\tau_{1}}\right)_{\#}(0,1)\right)=\psi(0,1)=b$. 
Finally, using (22), we have:

$$
\begin{aligned}
& \beta_{\#}(1,0)=\left(p_{1}\right)_{\#}(\varphi(1,0))=\left(p_{1}\right)_{\#}\left(a l_{\sigma}(a)\right)+\left(p_{1}\right)_{\#}(B, 0,0)=\left(p_{1}\right)_{\#}\left(a l_{\sigma}(a)\right), \text { and } \\
& \beta_{\#}(0,1)=\left(p_{1}\right)_{\#}(\varphi(0,1))=\left(p_{1}\right)_{\#}(b),
\end{aligned}
$$

from which we obtain (ii) and (iii). Conversely, suppose that there exist $a, b \in P_{2}\left(\mathbb{T}^{2}\right)$ such that (i), (ii) and (iii) are satisfied. We define $\psi: \pi_{1}\left(\mathbb{T}^{2}\right) \rightarrow P_{2}\left(\mathbb{T}^{2}\right)$ (resp. $\varphi: \pi_{1}\left(\mathbb{T}^{2}\right) \rightarrow B_{2}\left(\mathbb{T}^{2}\right)$ ) on the generators of $\pi_{1}\left(\mathbb{T}^{2}\right)$ by the correspondences (1) and (2) (resp. (3) and (4)). Since:

$$
\begin{aligned}
{[\varphi(1,0), \varphi(0,1)] } & =\left[a l_{\sigma}(a) \sigma^{2}, b\right]=a \sigma a \sigma^{-1} \sigma^{2} b \sigma^{-2} \sigma a^{-1} \sigma^{-1} a^{-1} b^{-1} \\
& =a l_{\sigma}\left(a l_{\sigma}(b) a^{-1}\right) a^{-1} b^{-1} \stackrel{(i)}{=} a l_{\sigma}\left(b a a^{-1}\right) a^{-1} b^{-1} \stackrel{(i)}{=} b a a^{-1} b^{-1}=\mathbb{1}, \text { and } \\
{[\psi(1,0), \psi(0,1)] } & =[a \sigma, b]=a \sigma b \sigma^{-1} a^{-1} b^{-1}=a l_{\sigma}(b) a^{-1} b^{-1} \stackrel{(i)}{=} b a a^{-1} b^{-1}=\mathbb{1} .
\end{aligned}
$$

these correspondences extend to homomorphisms. As in the first part of the proof, $\psi$ and $\varphi$ make the diagram (9) commute, so by Theorem [6 and (22), $\beta$ does not have the Borsuk-Ulam property with respect to $\tau_{1}$.

For each $\beta \in\left[\mathbb{T}^{2}, \mathbb{T}^{2}\right]$, let $\beta_{1,1}, \beta_{2,1}, \beta_{1,2}, \beta_{2,2} \in \mathbb{Z}$ be such that:

$$
\beta_{\#}(1,0)=\Delta(\beta)(1,0)=\left(\beta_{1,1}, \beta_{2,1}\right) \quad \text { and } \quad \beta_{\#}(0,1)=\Delta(\beta)(0,1)=\left(\beta_{1,2}, \beta_{2,2}\right) .
$$

Proposition 27. If $\beta \in\left[\mathbb{T}^{2}, \mathbb{T}^{2}\right]$ is a homotopy class then $\beta$ does not have the Borsuk-Ulam property with respect to $\tau_{1}$.

Proof. Let $r, s \in \mathbb{Z}$ and $i, j \in\{0,1\}$ be such that $\beta_{1,1}=2 r+i$ and $\beta_{2,1}=2 s+j$. With the identification of Theorem [14, let $a=\left(x^{i} y^{j}, r, s\right)$ and $b=\left(\mathbb{1}, \beta_{1,2}, \beta_{2,2}\right)$ of $P_{2}\left(\mathbb{T}^{2}\right)$. Let us show that these elements satisfy (i), (ii) and (iii) of Lemma 26, from which it will follow that $\beta$ does not have the Borsuk-Ulam property with respect to $\tau_{1}$. First:

$$
a l_{\sigma}(b)=\left(x^{i} y^{j}, r, s\right)\left(\mathbb{1}, \beta_{1,2}, \beta_{2,2}\right)=\left(\mathbb{1}, \beta_{1,2}, \beta_{2,2}\right)\left(x^{i} y^{j}, r, s\right)=b a,
$$

which yields (i). Using (12), we obtain:

$$
\left(p_{1}\right)_{\#}\left(a l_{\sigma}(a)\right)=(r, s)+(i, j)+(r, s)=\left(\beta_{1,1}, \beta_{2,1}\right)=\beta_{\#}(1,0),
$$

which proves (ii). Finally, we have:

$$
\left(p_{1}\right)_{\#}(b)=\left(\beta_{1,2}, \beta_{2,2}\right)=\beta_{\#}(0,1),
$$

whence (iii).

Proof of Theorem 1. The result is a consequence of Propositions 9, 25] and 27.

We now solve the Borsuk-Ulam problem with respect to the involution $\tau_{2}$. This case is more delicate than the previous one. We first prove some preliminary results.

Lemma 28. A free homotopy class $\beta \in\left[\mathbb{T}^{2}, \mathbb{T}^{2}\right]$ does not have the Borsuk-Ulam property with respect to $\tau_{2}$ if and only if there exist $a, b \in P_{2}\left(\mathbb{T}^{2}\right)$ such that:

(i) $a b l_{\sigma}(a)=b$.

(ii) $\beta_{\#}(1,0)=\left(p_{1}\right)_{\#}(a)$.

(iii) $\beta_{\#}(0,1)=\left(p_{1}\right)_{\#}\left(b l_{\sigma}(b)\right)$. 
Proof. We follow the proof of Lemma 26, replacing the homomorphism $\psi$ (resp. $\varphi$ ) by the homomorphism $\psi: \pi_{1}\left(\mathbb{K}^{2}\right) \rightarrow B_{2}\left(\mathbb{T}^{2}\right)$ (resp. $\varphi: \pi_{1}\left(\mathbb{T}^{2}\right) \rightarrow P_{2}\left(\mathbb{T}^{2}\right)$ ) defined on the generators of $\pi_{1}\left(\mathbb{K}^{2}\right)$ (resp. of $\left.\pi_{1}\left(\mathbb{T}^{2}\right)\right)$ by $\psi(1,0)=a$ and $\psi(0,1)=b \sigma\left(\operatorname{resp} . \varphi(1,0)=a\right.$ and $\left.\varphi(0,1)=b l_{\sigma}(b) \sigma^{2}\right)$. The details are left as an exercise.

Proposition 29. Suppose that $\beta \in\left[\mathbb{T}^{2}, \mathbb{T}^{2}\right]$ satisfies one of the following conditions:

1. at least one of $\beta_{1,2}$ and $\beta_{2,2}$ is odd.

2. $\left(\beta_{1,1}, \beta_{2,1}\right)=(0,0)$ and $\beta_{1,2}$ and $\beta_{2,2}$ are both even.

Then $\beta$ does not have the Borsuk-Ulam property with respect to $\tau_{2}$.

Proof. Assume that condition 1 of the statement is satisfied. Suppose first that $\beta_{1,2}$ and $\beta_{2,2}$ are odd. Let $r, s \in \mathbb{Z}$ be such that $\beta_{1,2}=2 r+1$ and $\beta_{2,2}=2 s+1$. With the identification of Theorem 14, consider the elements $a=\left(x^{-2 \beta_{1,1}} y^{-2 \beta_{2,1}}, \beta_{1,1}, \beta_{1,2}\right)$ and $b=\left(y^{1+2 \beta_{2,1}} x^{-1}, r+1, s-\beta_{2,1}\right)$ of $P_{2}\left(\mathbb{T}^{2}\right)$. We will show that $a$ and $b$ satisfy (i), (ii) and (iii) of Lemma 28, from which it will follow that $\beta$ does not have the Borsuk-Ulam property with respect to $\tau_{2}$. Using (12) we obtain:

$$
\begin{aligned}
a b l_{\sigma}(a) & =\left(x^{-2 \beta_{1,1}} y^{-2 \beta_{2,1}}, \beta_{1,1}, \beta_{1,2}\right)\left(y^{1+2 \beta_{2,1}} x^{-1}, r+1, s-\beta_{2,1}\right) l_{\sigma}\left(x^{-2 \beta_{1,1}} y^{-2 \beta_{2,1}}, \beta_{1,1}, \beta_{1,2}\right) \\
& =\left(x^{-2 \beta_{1,1}} y x^{-1}, \beta_{1,1}+r+1, s\right)\left(x y^{-1} x^{2 \beta_{1,1}} y^{2 \beta_{1,2}} y x^{-1},-\beta_{1,1},-\beta_{1,2}\right) \\
& =\left(y^{1+2 \beta_{2,1}} x^{-1}, r+1, s-\beta_{2,1}\right)=b
\end{aligned}
$$

and hence condition (i) is satisfied. Further:

$$
\begin{aligned}
\left(p_{1}\right)_{\#}(a) & =\left(\beta_{1,1}, \beta_{1,2}\right) \text { and } \\
\left(p_{1}\right)_{\#}\left(b l_{\sigma}(b)\right) & =\left(r+1, s-\beta_{2,1}\right)+\left(-1,1+2 \beta_{2,1}\right)+\left(r+1, s-\beta_{2,1}\right)=\left(\beta_{1,2}, \beta_{2,2}\right),
\end{aligned}
$$

from which it follows that conditions (ii) and (iii) are satisfied, and hence $\beta$ does not have the Borsuk-Ulam property with respect to $\tau_{2}$. The remaining cases are analogous, and we just provide the elements $a$ and $b$ of $P_{2}\left(\mathbb{T}^{2}\right)$ in each case that satisfy (i), (ii) and (iii) of Lemma 28, the details being left as an exercise. If $\beta_{1,2}$ is odd and $\beta_{2,2}$ is even, we take $\beta_{1,2}=2 r+1$ and $\beta_{2,2}=2 s$, where $r, s \in \mathbb{Z}$, and $a=\left(x^{-\beta_{1,1}} y^{-2 \beta_{2,1}-1} x^{-\beta_{1,1}} y, \beta_{1,1}, \beta_{2,1}\right)$ and $b=\left(x^{-1}, r+1, s\right)$. If $\beta_{1,2}$ is even and $\beta_{2,2}$ is odd, we set $\beta_{1,2}=2 r$ and $\beta_{2,2}=2 s+1$, where $r, s \in \mathbb{Z}$, and $a=\left(x^{-2 \beta_{1,1}+1} y^{-\beta_{2,1}} x^{-1} y^{-\beta_{2,1}}, \beta_{1,1}, \beta_{2,1}\right)$ and $b=\left(x^{-2 \beta_{1,1}+1} y x^{-1}, \beta_{1,1}+r, s\right)$. Finally, if $\beta$ satisfies condition 2 of the statement, we take $\beta_{1,2}=2 r$ and $\beta_{2,2}=2 s$, where $r, s \in \mathbb{Z}$, and $a=(\mathbb{1}, 0,0)$ and $b=(\mathbb{1}, r, s)$.

Note that the case where $\left(\beta_{1,1}, \beta_{2,1}\right) \neq(0,0)$ and $\beta_{1,2}$ and $\beta_{2,2}$ are even is not addressed by Proposition 29. Before analysing this situation, let $z=z(x, y)$ be a reduced word in $F(x, y)$. Then $z$ is called a palindrome if $z(x, y)=z\left(x^{-1}, y^{-1}\right)^{-1}$. This means that $z$ reads the same backwards as forwards. If $z=w_{1}^{\epsilon_{1}} \cdots w_{m}^{\epsilon_{m}}$, where for all $i=1, \ldots, m, w_{i} \in\{x, y\}$ and $\epsilon_{i} \in \mathbb{Z}$, the length $\ell(z)$ of $z$ is defined to be equal to $\sum_{i=1}^{m}\left|\epsilon_{i}\right|$. We now prove two technical lemmas.

Lemma 30. If $z=z(x, y) \in F(x, y)$ is a palindrome, then $|z|_{x}$ or $|z|_{y}$ is even.

Proof. Let $z=z(x, y)$ be a reduced word in $F(x, y)$. If $\ell(z)=0$ or $\ell(z)=1$ then $z$ is trivial or $z \in\left\{x, x^{-1}, y, y^{-1}\right\}$ respectively, and at least one of $|z|_{x}$ and $|z|_{y}$ is zero. If $\ell(z)=2$ then $z \in\left\{x^{2 a}, y^{2 a}, x^{a} y^{b}, y^{b} x^{a} \mid a, b \in\{-1,1\}\right\}$. Since $z$ is a palindrome, $z=x^{2 a}$ or $z=y^{2 b}$ and the result follows. So suppose by induction that the result holds for palindromes of length less than or equal to $r$, where $r \geq 2$, and let $z$ be a palindrome such that $\ell(z)=r+1$. By definition of palindrome, we have $z=k z^{\prime} k$, where $k \in\left\{x, x^{-1}, y, y^{-1}\right\}, z^{\prime} \in F(x, y)$ and $k z^{\prime} k$ is in reduced form. Then $\ell\left(z^{\prime}\right)=r-2$ and

$$
k z^{\prime} k=z=z\left(x^{-1}, y^{-1}\right)^{-1}=\left(k^{-1} z^{\prime}\left(x^{-1}, y^{-1}\right) k^{-1}\right)^{-1}=k z^{\prime}\left(x^{-1}, y^{-1}\right)^{-1} k .
$$

Thus $z^{\prime}$ is a palindrome, and $\left|z^{\prime}\right|_{x}$ or $\left|z^{\prime}\right|_{y}$ is even by induction. The result follows using the fact that $|z|_{g} \equiv\left|z^{\prime}\right|_{g} \bmod 2$ for all $g \in\{x, y\}$. 
Lemma 31. Let $z, w \in F(x, y)$ be such that $w$ is non trivial, $|w|_{x}$ and $|w|_{y}$ are even and

$$
z w z^{-1}=w\left(x^{-1}, y^{-1}\right)^{-1} .
$$

Then $|z|_{x}$ or $|z|_{y}$ is even.

Proof. The result is immediate if $z$ is trivial. So assume that $z$ is non trivial. Without loss of generality, we may suppose that $z$ and $w$ are each written in reduced form. Since $\ell(w)=\ell\left(w\left(x^{-1}, y^{-1}\right)^{-1}\right)$, there must be cancellation on the left-hand side of equation (23). We are interested in the parity of $|z|_{x}$ and $|z|_{y}$. The following operations show that, while respecting the hypotheses of the statement of the lemma, we may modify $z$ and $w$ so as to control better the possible cancellations in (23). In what follows, let $g \in\{x, y\}$.

Step 1: We may assume that cancellation occurs at $z w$ or at $w z^{-1}$, but not at both. For suppose that cancellation occurs at $z w$ and at $w z^{-1}$. Then $z=z_{1} k$ and $w=k^{-1} w_{1} k$ in reduced form for some $k \in\left\{x, x^{-1}, y, y^{-1}\right\}$ and some $z_{1}, w_{1} \in F(x, y)$. Now $w_{1}$ is non trivial and $\left|w_{1}\right|_{x}$ and $\left|w_{1}\right|_{y}$ are even. Also, $\left(k^{-1} z_{1}\right) w_{1}\left(k^{-1} z_{1}\right)^{-1}=w_{1}\left(x^{-1}, y^{-1}\right)^{-1}$ by (23) . This equation is of the same nature as that of (23), and it is easy to see that $\left|k^{-1} z_{1}\right|_{g} \equiv|z|_{g} \bmod 2$. If cancellation occurs at $\left(k^{-1} z_{1}\right) w_{1}$ and at $w_{1}\left(k^{-1} z_{1}\right)^{-1}$, we repeat the argument. After a finite number of operations (possibly zero, and at most $\ell(z)$ times), we obtain elements $z^{\prime}, w^{\prime} \in F(x, y)$ in reduced form for which $\left|z^{\prime}\right|_{g} \equiv|z|_{g} \bmod 2$, that continue to satisfy the hypotheses of the lemma, and such that any cancellation in $z^{\prime} w^{\prime} z^{\prime-1}$ occurs either at $z w^{\prime}$ or at $z w^{\prime-1}$, but not at both.

Step 2: We may assume that cancellation occurs at $z w$. For if cancellation occurs at $w z^{-1}$, by setting $w^{\prime}=w^{-1}$, the elements $z, w^{\prime} \in F(x, y)$ satisfy the hypotheses of the lemma, and cancellation occurs at $z w^{\prime}$.

Step 3: We may assume that $z$ cannot be written in reduced form $z^{\prime} w^{\varepsilon}$, where $\varepsilon \in\{-1,1\}$. For if $z=z_{1} w^{\varepsilon_{1}}$ in reduced form for some $z_{1} \in F(x, y)$ and some $\varepsilon_{1} \in\{-1,1\}$, then $z_{1} w z_{1}^{-1}=w\left(x^{-1}, y^{-1}\right)^{-1}$ by (23). Hence the elements $z_{1}, w \in F(x, y)$ satisfy the hypotheses of the lemma, and $|z|_{g}=$ $\left|z_{1}\right|_{g}+\varepsilon_{1}|w|_{g} \equiv\left|z_{1}\right|_{g} \bmod 2$. If $z_{1}$ may be writen in reduced form $z_{2} w^{\varepsilon_{2}}$, for some $z_{2} \in F(x, y)$ and some $\varepsilon_{2} \in\{-1,1\}$, we repeat the argument. After a finite number of operations, we obtain an element $z^{\prime} \in F(x, y)$ such that $z^{\prime}$ and $w$ satisfy the hypotheses of the lemma, $z^{\prime}$ cannot be written in reduced form $\tilde{z} w^{\varepsilon}$ for some $\tilde{z} \in F(x, y)$ and some $\varepsilon \in\{-1,1\}$, and $|z|_{g} \equiv\left|z^{\prime}\right|_{g} \bmod 2$.

After applying steps 1,2 and 3 as many times as necessary, we may suppose without loss of generality that there exist elements $z_{1}, z_{2}, w_{1}, w_{2} \in F(x, y)$ such that $z=z_{1} z_{2}$ and $w=w_{1} w_{2}$ in reduced form, $w_{1}$ and $w_{2}$ are non trivial, $w_{1}=z_{2}^{-1}$, and there is no cancellation either at $z_{1} w_{2}$ or at $w_{2} z_{2}^{-1}$. By (23), we have:

$$
z_{1} w_{2} w_{1} z_{1}^{-1}=w_{2}\left(x^{-1}, y^{-1}\right)^{-1} w_{1}\left(x^{-1}, y^{-1}\right)^{-1}
$$

By hypothesis, there is no cancellation in $z_{1} w_{2} w_{1} z_{1}^{-1}$. But $\ell\left(w_{2} w_{1}\right)=\ell\left(w_{1} w_{2}\right)$, which in turn is equal to $\ell\left(w_{2}\left(x^{-1}, y^{-1}\right)^{-1} w_{1}\left(x^{-1}, y^{-1}\right)^{-1}\right)$. So by (24),$z_{1}$ is trivial and $w_{i}=w_{i}\left(x^{-1}, y^{-1}\right)^{-1}$, for all $i \in\{1,2\}$. Thus $z=w_{1}^{-1}$, and therefore it is a palindrome. The result then follows from Lemma 30 .

Proposition 32. Suppose that $\beta \in\left[\mathbb{T}^{2}, \mathbb{T}^{2}\right]$ satisfies $\left(\beta_{1,1}, \beta_{2,1}\right) \neq(0,0)$, and $\beta_{1,2}$ and $\beta_{2,2}$ are even. Then $\beta$ has the Borsuk-Ulam property with respect to $\tau_{2}$.

Proof. We argue by contradiction. Suppose that $\beta$ does not have the Borsuk-Ulam property with respect to $\tau_{2}$. By Lemma 28, there exist $a=\left(w(x, y), r_{a}, s_{a}\right)$ and $b=\left(z(x, y), r_{b}, s_{b}\right)$ such that $a, b$ 
and $\beta_{\#}$ satisfy items (i), (ii) and (iii). By (ii), we have $\left(r_{a}, s_{a}\right)=\left(\beta_{1,1}, \beta_{2,1}\right)$. Using (iii), (12) implies that:

$$
\left(\beta_{1,2}, \beta_{2,2}\right)=\left(p_{1}\right)_{\#}\left(b l_{\sigma}(b)\right)=\left(|z|_{x}+2 r_{b},|z|_{y}+2 s_{b}\right),
$$

and we see that $|z|_{x}$ and $|z|_{y}$ are even. Further:

$$
\begin{aligned}
a b l_{\sigma}(a) & =\left(w, \beta_{1,1}, \beta_{2,1}\right)\left(z, r_{b}, s_{b}\right)\left(x y^{-1} w\left(x^{-1}, y^{-1}\right) y x^{-1},|w|_{x}+\beta_{1,1},|w|_{y}+\beta_{2,1}\right) \\
& =\left(w z x y^{-1} w\left(x^{-1}, y^{-1}\right) y x^{-1}, 2 \beta_{1,1}+|w|_{x}+r_{b}, 2 \beta_{2,1}+|w|_{y}+s_{b}\right) .
\end{aligned}
$$

By condition (i), it follows that $|w|_{x}=-2 \beta_{1,1}$ and $|w|_{y}=-2 \beta_{2,1}$, and thus $w$ is non trivial. Furthermore, $w z x y^{-1} w\left(x^{-1}, y^{-1}\right) y x^{-1}=z$ by $(\mathrm{i})$, and so:

$$
\left(y x^{-1} z^{-1}\right) w\left(y x^{-1} z^{-1}\right)^{-1}=w\left(x^{-1}, y^{-1}\right)^{-1} .
$$

By Lemma 31, we see that $\left|y x^{-1} z^{-1}\right|_{x}=-1-|z|_{x}$ or $\left|y x^{-1} z^{-1}\right|_{y}=1-|z|_{y}$ is even, or equivalently, that $|z|_{x}$ or $|z|_{y}$ is odd. But this contradicts the fact that $|z|_{x}$ and $|z|_{y}$ are even. Thus $\beta$ has the Borsuk-Ulam property with respect to $\tau_{2}$ as required.

Proof of Theorem 2 . The result follows directly from Propositions 29 and 32.

\section{Proof of Theorem 3}

The purpose of this section is to prove Theorem 3 , We identify $\pi_{1}\left(\mathbb{K}^{2}\right)$ with $\mathbb{Z} \rtimes \mathbb{Z}$ as in Section 4 . Consider the following short exact sequence:

$$
\begin{gathered}
1 \longrightarrow \pi_{1}\left(\mathbb{K}^{2}\right) \stackrel{i_{3}}{\longrightarrow} \pi_{1}\left(\mathbb{K}^{2}\right) \stackrel{\theta_{3}}{\longrightarrow} \mathbb{Z}_{2} \longrightarrow 1 \\
i_{3}:\left\{\begin{array}{l}
(1,0) \longmapsto(2,0) \\
(0,1) \longmapsto(0,1)
\end{array} \theta_{3}:\left\{\begin{array}{l}
(1,0) \longmapsto \overline{1} \\
(0,1) \longmapsto \overline{0} .
\end{array}\right.\right.
\end{gathered}
$$

By covering space theory, there exists a double covering $c_{3}: \mathbb{K}^{2} \rightarrow \mathbb{K}^{2}$ such that the induced homomorphism on the fundamental group is $i_{3}$. Let $\tau_{3}: \mathbb{K}^{2} \rightarrow \mathbb{K}^{2}$ be the non-identity deck transformation associated with $c_{3}$. Then $\tau_{3}$ is a free involution, and (25) is the short exact sequence induced by $\tau_{3}$. Further, $\tau_{3}$ lifts to a homeomorphism $\widehat{\tau}_{3}: \mathbb{R}^{2} \rightarrow \mathbb{R}^{2}$, where $\widehat{\tau}_{3}(x, y)=\left(x, y+\frac{1}{2}\right)$ for all $(x, y) \in \mathbb{R}^{2}$. As the following result shows, up to the equivalence relation defined just after Proposition 9 , this is the only free involution on the Klein bottle.

Proposition 33. Let $\tau: \mathbb{K}^{2} \rightarrow \mathbb{K}^{2}$ be a free involution. Then $\tau$ is equivalent to $\tau_{3}$.

Proof. Since the natural projection $p_{\tau}: \mathbb{K}^{2} \rightarrow \mathbb{K}_{\tau}^{2}$ is a covering, $\mathbb{K}_{\tau}^{2}$ is homeomorphic to the Klein bottle, and the short exact sequence induced by $\tau$ is of the following form:

$$
1 \longrightarrow \pi_{1}\left(\mathbb{K}^{2}\right)=\mathbb{Z} \rtimes \mathbb{Z} \stackrel{\left(p_{\tau}\right)_{\#}}{\longrightarrow} \pi_{1}\left(\mathbb{K}_{\tau}^{2}\right) \stackrel{\theta_{\tau}}{\longrightarrow} \mathbb{Z}_{2} \longrightarrow 1
$$

By Remark 17 we have an isomorphism $\gamma: \mathbb{Z} \rtimes \mathbb{Z} \rightarrow \pi_{1}\left(\mathbb{K}_{\tau}^{2}\right)$. The composition $\theta_{\tau} \circ \gamma$ is different from the homomorphism $\theta_{2}$ defined in (19). Indeed, if $\theta_{\tau} \circ \gamma=\theta_{2}$, then $\pi_{1}\left(\mathbb{K}^{2}\right) \cong \operatorname{ker} \theta_{\tau} \circ \gamma=$ ker $\theta_{2} \cong \pi_{1}\left(\mathbb{T}^{2}\right)$, which is absurd. By [8, Proposition 32], there exists an isomorphism $\psi: \pi_{1}\left(\mathbb{K}^{2}\right) \rightarrow$ $\pi_{1}\left(\mathbb{K}_{\tau}^{2}\right)$ such that $\theta_{3}=\theta_{\tau} \circ \psi$. Comparing (25) and (26) and using exactness and the 5-Lemma, it follows that $\psi$ induces an isomorphism $\varphi: \pi_{1}\left(\mathbb{K}^{2}\right) \rightarrow \pi_{1}\left(\mathbb{K}^{2}\right)$ such that $\left(p_{\tau}\right)_{\#} \circ \varphi=\psi \circ i_{3}$. So the exact sequences (25) and (26) are equivalent, and we conclude that $\tau$ and $\tau_{1}$ are equivalent by Proposition 10 . 
By Theorem 4, the following diagram (we simplify the notation):

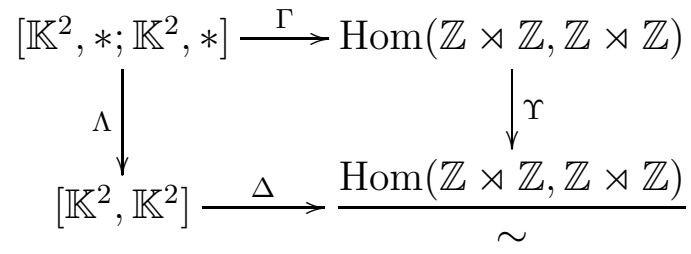

is commutative, where the maps $\Gamma$ and $\Delta$ are bijections and the maps $\Lambda$ and $\Upsilon$ are surjective. So, in order to determine the sets $\left[\mathbb{K}^{2}, * ; \mathbb{K}^{2}, *\right]$ and $\left[\mathbb{K}^{2}, \mathbb{K}^{2}\right]$, we will describe the elements of the sets $\operatorname{Hom}(\mathbb{Z} \rtimes \mathbb{Z}, \mathbb{Z} \rtimes \mathbb{Z})$ and $\frac{\operatorname{Hom}(\mathbb{Z} \rtimes \mathbb{Z}, \mathbb{Z} \rtimes \mathbb{Z})}{\sim}$.

Remark 34. By [10, Lemma 3.1], for each $h \in \operatorname{Hom}(\mathbb{Z} \rtimes \mathbb{Z}, \mathbb{Z} \rtimes \mathbb{Z})$, we have the following two possibilities for the images of the generators of $\mathbb{Z} \rtimes \mathbb{Z}$ :

- $h(1,0)=\left(r_{1}, 0\right)$ and $h(0,1)=\left(r_{2}, 2 s+1\right)$ for some $r_{1}, r_{2}, s \in \mathbb{Z}$. In this case, we say that $h$ is of type A.

- $h(1,0)=(0,0)$ and $h(0,1)=(r, 2 s)$ for some $r, s \in \mathbb{Z}$. In this case, we say that $h$ is of type $B$.

Proposition 35. Let $h \in \operatorname{Hom}(\mathbb{Z} \rtimes \mathbb{Z}, \mathbb{Z} \rtimes \mathbb{Z})$.

(a) If $h$ is of type $A$ then there exist unique integers $i \in\{0,1\}, r, s \in \mathbb{Z}$, where $r \geq 0$, such that $h$ is conjugate to the homomorphism given by $\left\{\begin{array}{l}(1,0) \longmapsto(r, 0) \\ (0,1) \longmapsto(i, 2 s+1) \text {. }\end{array}\right.$

(b) If $h$ is of type $B$ then there exist unique integers $r, s \in \mathbb{Z}$, where $r \geq 0$, such that $h$ is conjugate to the homomorphism given by $\left\{\begin{array}{l}(1,0) \longmapsto(0,0) \\ (0,1) \longmapsto(r, 2 s) .\end{array}\right.$

Proof. If $h: \mathbb{Z} \rtimes \mathbb{Z} \rightarrow \mathbb{Z} \rtimes \mathbb{Z}$ is of Type A, then conjugating $h$ by the element $(a, b) \in \mathbb{Z} \rtimes \mathbb{Z}$, we obtain a homomorphism $h^{\prime}: \mathbb{Z} \rtimes \mathbb{Z} \rightarrow \mathbb{Z} \rtimes \mathbb{Z}$ that depends on $r_{1}$ and $r_{2}$ as follows:

- if $r_{1} \geq 0$ (resp. $\left.r_{1}<0\right)$ and $r_{2}$ is even, let $a=-\frac{r_{2}}{2}$ and $b=0$ (resp. $a=\frac{r_{2}}{2}$ and $\left.b=1\right)$. Then $h^{\prime}:\left\{\begin{array}{l}(1,0) \longmapsto\left(\left|r_{1}\right|, 0\right) \\ (0,1) \longmapsto(0,2 s+1) .\end{array}\right.$

- if $r_{1} \geq 0$ (resp. $\left.r_{1}<0\right)$ and $r_{2}$ is odd, let $a=\frac{-r_{2}+1}{2}$ and $b=0$ (resp. $a=\frac{r_{2}+1}{2}$ and $\left.b=1\right)$. Then $h^{\prime}:\left\{\begin{array}{l}(1,0) \longmapsto\left(\left|r_{1}\right|, 0\right) \\ (0,1) \longmapsto(1,2 s+1) .\end{array}\right.$

If $h: \mathbb{Z} \rtimes \mathbb{Z} \rightarrow \mathbb{Z} \rtimes \mathbb{Z}$ is of Type B, then conjugating $h$ by $(0,1)$, we obtain a homomorphism $h^{\prime}: \mathbb{Z} \rtimes \mathbb{Z} \rightarrow$ $\mathbb{Z} \rtimes \mathbb{Z}$ defined by: $h^{\prime}:\left\{\begin{array}{l}(1,0) \longmapsto(0,0) \\ (0,1) \longmapsto(-r, 2 s) .\end{array} \quad\right.$ We conclude that each element $h \in \operatorname{Hom}(\mathbb{Z} \rtimes \mathbb{Z}, \mathbb{Z} \rtimes \mathbb{Z})$ is conjugate to one of the homomorphisms given in the statement of the proposition. Further, if $h_{1}, h_{2}: \mathbb{Z} \rtimes \mathbb{Z} \rightarrow \mathbb{Z} \rtimes \mathbb{Z}$ are two such homomorphisms, then we leave it as an exercise to show that if they are distinct, then they are non conjugate, and the result follows.

Remark 36. By diagram (27) and Proposition 35, there exists a bijection between the set $\left[\mathbb{K}^{2}, \mathbb{K}^{2}\right]$ and the subset of $\operatorname{Hom}(\mathbb{Z} \rtimes \mathbb{Z}, \mathbb{Z} \rtimes \mathbb{Z})$ whose elements are described in parts (a) and (b) of Proposition [35. By abuse of notation, for each $\beta \in\left[\mathbb{K}^{2}, \mathbb{K}^{2}\right]$, the image of $\beta$ under the above bijection shall be denoted by $\beta_{\#}$. 
We now solve the Borsuk-Ulam problem for homotopy classes with respect to involution $\tau_{3}$. As in Lemmas 26 and 28, we have the following result:

Lemma 37. A free homotopy class $\beta \in\left[\mathbb{K}^{2}, \mathbb{K}^{2}\right]$ does not have the Borsuk-Ulam property with respect to $\tau_{3}$ if and only if there exist pure braids $a, b \in P_{2}\left(\mathbb{K}^{2}\right)$ such that:

(i) $l_{\sigma}(a) l_{\sigma}(b) \sigma^{2} a=b$.

(ii) $\beta_{\#}(1,0)=\left(p_{1}\right)_{\#}\left(l_{\sigma}(a) a\right)$.

(iii) $\beta_{\#}(0,1)=\left(p_{1}\right)_{\#}(b)$.

Proof. First, suppose that $\beta$ does not have the Borsuk-Ulam property with respect to $\tau_{3}$. By (27) and Proposition 7, there exists a pointed homotopy class $\alpha$ that does not have the Borsuk-Ulam property such that $\alpha_{\mathcal{F}}=\Lambda(\alpha)=\beta$. By Proposition [6, there exist homomorphisms $\varphi: \pi_{1}\left(\mathbb{K}^{2}\right) \rightarrow P_{2}\left(\mathbb{K}^{2}\right)$ and $\psi: \pi_{1}\left(\mathbb{K}^{2}\right) \rightarrow B_{2}\left(\mathbb{K}^{2}\right)$ that makes diagram (9) commute. By (25), $\psi(1,0) \in B_{2}\left(\mathbb{K}^{2}\right) \backslash P_{2}\left(\mathbb{K}^{2}\right)$ and $\psi(0,1) \in P_{2}\left(\mathbb{K}^{2}\right)$. From the short exact sequence (8) and Theorem 15, there exist $a, b \in P_{2}\left(\mathbb{T}^{2}\right)$ such that:

(1) $\psi(1,0)=\sigma a$.

(2) $\psi(0,1)=b$.

By Remark[17, we have $\mathbb{1}=[\psi(1,0), \psi(0,1)]^{\prime}=\sigma a b \sigma a b^{-1}$, which is equivalent to $\left(\sigma a \sigma^{-1}\right)\left(\sigma b \sigma^{-1}\right) \sigma^{2} a=$ $b$, and which yields (i). The commutativity of (9) gives rise to the following relations:

(3) $\varphi(1,0)=\psi\left(\left(p_{\tau_{1}}\right)_{\#}(1,0)\right)=\psi(2,0)=(\sigma a)^{2}=l_{\sigma}(a) \sigma^{2} a$.

(4) $\varphi(0,1)=\psi\left(\left(p_{\tau_{1}}\right)_{\#}(0,1)\right)=\psi(0,1)=b$.

Finally, $\beta_{\#}(1,0)=\left(p_{1}\right)_{\#}(\varphi(1,0))=\left(p_{1}\right)_{\#}\left(l_{\sigma}(a) \sigma^{2} a\right)=\left(p_{1}\right)_{\#}\left(l_{\sigma}(a) a\right)$ and $\beta_{\#}(0,1)=\left(p_{1}\right)_{\#}(\varphi(0,1))=$ $\left(p_{1}\right)_{\#}(b)$ by (27), from which we obtain (ii) and (iii). Conversely, suppose that there exist $a, b \in$ $P_{2}\left(\mathbb{K}^{2}\right)$ such that conditions (i), (ii) and (iii) are satisfied. We define $\psi: \pi_{1}\left(\mathbb{K}^{2}\right) \rightarrow P_{2}\left(\mathbb{K}^{2}\right)$ (resp. $\varphi: \pi_{1}\left(\mathbb{K}^{2}\right) \rightarrow B_{2}\left(\mathbb{K}^{2}\right)$ ) on the generators of $\pi_{1}\left(\mathbb{K}^{2}\right)$ by equations (1) and (2) (resp. (3) and (4)). These maps extend to homomorphisms because:

$$
\begin{aligned}
{[\varphi(1,0), \varphi(0,1)]^{\prime} } & =l_{\sigma}(a) \sigma^{2} a b l_{\sigma}(a) \sigma^{2} a b^{-1}=l_{\sigma}\left(a l_{\sigma}(a) l_{\sigma}(b) \sigma^{2} a\right) \sigma^{2} a b^{-1} \stackrel{(\mathrm{i})}{=} l_{\sigma}(a b) \sigma^{2} a b^{-1} \\
& =l_{\sigma}(a) l_{\sigma}(b) \sigma^{2} a b^{-1} \stackrel{(\mathrm{i})}{=} \mathbb{1} \text { and } \\
{[\psi(1,0), \psi(0,1)]^{\prime} } & =\sigma a b \sigma a b^{-1}=l_{\sigma}(a) l_{\sigma}(b) \sigma^{2} a b^{-1} \stackrel{(\mathrm{i})}{=} \mathbb{1} .
\end{aligned}
$$

By a calculation similar to that given in the first part of the proof, we see that diagram (9) is commutative. So by Proposition [6] and (27), $\beta$ does not have the Borsuk-Ulam property with respect to $\tau_{3}$.

Proposition 38. With the notation of Proposition 35 and Remark [36, let $\beta \in\left[\mathbb{K}^{2}, \mathbb{K}^{2}\right]$ be such that the homomorphism $\beta_{\#}: \mathbb{Z} \rtimes \mathbb{Z} \rightarrow \mathbb{Z} \rtimes \mathbb{Z}$ is of Type $A$. Then $\beta$ does not have the Borsuk-Ulam property with respect to $\tau_{3}$.

Proof. First, suppose that $r$ is even. Let $m \in \mathbb{Z}$ be such that $r=2 m$. With the notation of Theorem 23, consider the elements $a=(\mathbb{1} ; m, 0)$ and $b=(B ; i, 2 s+1)$ of $P_{2}\left(\mathbb{K}^{2}\right)$. Let us show that 
these elements satisfy the hypotheses (i), (ii) and (iii) of Lemma 37, from which it will follow that $\beta$ does not have the Borsuk-Ulam property with respect to $\tau_{3}$. Using Theorem 24, we have:

$$
\begin{aligned}
l_{\sigma}(a) l_{\sigma}(b) \sigma^{2} a & =l_{\sigma}(\mathbb{1} ; m, 0) l_{\sigma}(B ; i, 2 s+1)(B ; 0,0)(\mathbb{1} ; m, 0) \\
& =(\mathbb{1} ; m, 0) l_{\sigma}(B ; 0,0) l_{\sigma}(\mathbb{1} ; i, 0) l_{\sigma}(\mathbb{1} ; 0,2 s+1)(B ; m, 0) \\
& =(\mathbb{1} ; m, 0)(B ; 0,0)(\mathbb{1} ; i, 0)(B ; 0,2 s+1)(B ; m, 0) \\
& =(\theta(m, 0)(B) ; m+i, 0)(B \theta(0,2 s+1)(B) ;-m, 2 s+1) \\
& =(B ; m+i, 0)(\mathbb{1} ;-m, 2 s+1)=(B ; i, 2 s+1)=b,
\end{aligned}
$$

and hence condition (i) is satisfied. Further,

$$
\begin{aligned}
\left(p_{1}\right)_{\#}\left(l_{\sigma}(a) a\right) & =\left(p_{1}\right)_{\#}\left(l_{\sigma}(\mathbb{1} ; m, 0)(\mathbb{1} ; m, 0)\right)=\left(p_{1}\right)_{\#}((\mathbb{1} ; m, 0)(\mathbb{1} ; m, 0))=(2 m, 0) \\
& =(r, 0)=\beta_{\#}(1,0), \text { and } \\
\left(p_{1}\right)_{\#}(b) & =\left(p_{1}\right)_{\#}(B ; i, 2 s+1)=(i, 2 s+1)=\beta_{\#}(0,1)
\end{aligned}
$$

and thus conditions (ii) and (iii) are also satisfied.

Now suppose that $r$ is odd, and let $m \in \mathbb{Z}$ be such that $r=2 m+1$. Consider the elements $a=\left(u B^{-m} ; m, 0\right)$ and $b=\left(u^{-1} B^{\delta_{(i+1)}} ; i, 2 s+1\right)$ of $P_{2}\left(\mathbb{K}^{2}\right)$. The conclusion follows in a manner similar to that of the previous case, and the details are left as an exercise.

Proposition 39. With the notation of Proposition 35 and Remark [36, let $\beta \in\left[\mathbb{K}^{2}, \mathbb{K}^{2}\right]$ be such that the homomorphism $\beta_{\#}: \mathbb{Z} \rtimes \mathbb{Z} \rightarrow \mathbb{Z} \rtimes \mathbb{Z}$ is of Type $B$. Then $\beta$ has the Borsuk-Ulam property with respect to $\tau_{3}$.

Proof. We argue by contradiction. Suppose that $\beta$ does not have the Borsuk-Ulam property with respect to $\tau_{3}$. By Proposition 7 and diagram (27), there exists a pointed map $f:\left(\mathbb{K}^{2}, *\right) \rightarrow\left(\mathbb{K}^{2}, *\right)$ such that $f_{\#}=\beta_{\#}$ and $f\left(\tau_{3}(x)\right) \neq f(x)$ for all $x \in \mathbb{K}^{2}$. Notice that the image of $f_{\#}$ is contained in the image of the homomorphism $i_{2}: \pi_{1}\left(\mathbb{T}^{2}\right) \rightarrow \pi_{1}\left(\mathbb{K}^{2}\right)$ that is defined in (19). Recall that $c_{2}: \mathbb{T}^{2} \rightarrow \mathbb{K}^{2}$ is the double covering such that $\left(c_{2}\right)_{\#}=i_{2}$. So, by covering space theory, there exists a pointed map $\tilde{f}:\left(\mathbb{K}^{2}, *\right) \rightarrow\left(\mathbb{T}^{2}, *\right)$ such that $c_{2} \circ \tilde{f}=f$. Therefore, the map $\tilde{f}$ satisfies $\tilde{f}\left(\tau_{3}(x)\right) \neq \tilde{f}(x)$ for all $x \in \mathbb{K}^{2}$, which implies that the triple $\left(\mathbb{K}^{2}, \tau_{3} ; \mathbb{T}^{2}\right)$ does not have the Borsuk-Ulam property. But this yields a contradiction using Remark 17, the short exact sequence (25) and [8, Proposition 10]. The result then follows.

We are now able to prove the final main result of this paper.

Proof of Theorem 3. Using arguments similar to those of Proposition 39 and the notation of Remark [36, a homotopy class $\beta \in\left[\mathbb{K}^{2}, \mathbb{K}^{2}\right]$ lifts to the torus if and only if the homomorphism $\beta_{\#}$ : $\mathbb{Z} \rtimes \mathbb{Z} \rightarrow \mathbb{Z} \rtimes \mathbb{Z}$ is of Type B. Further, if $H: \mathbb{K}^{2} \rightarrow \mathbb{K}^{2}$ is a homeomorphism and there exist maps $f_{1}, f_{2}: \mathbb{K}^{2} \rightarrow \mathbb{K}^{2}$ such that $f_{2}=f_{1} \circ H^{-1}$, then $f_{1}$ lifts to the torus if and only if $f_{2}$ lifts to the torus. The result then follows easily from Propositions 9 , 33, 38, and 39,

\section{Acknowledgements}

Part of this work is contained in the Ph.D. thesis [11] of the third author who was supported by CNPq project $\mathrm{n}^{\circ} 140836$ and Capes/COFECUB project $\mathrm{n}^{\circ} 12693 / 13-8$. The first and second authors were partially supported by CNRS/FAPESP project number (2014/50131-7: Brazil; 2014/226555: France). The second author wishes to thank the 'Réseau Franco-Brésilien en Mathématiques' for financial support during his visit to the Instituto de Matemática e Estatística, Universidade de São Paulo, from the $9^{\text {th }}$ of July to the $1^{\text {st }}$ of August 2016. 


\section{References}

[1] P. Bellingeri, On presentations of surface braid groups, J. Algebra 274 (2004), 543-563.

[2] P. Bellingeri, S. Gervais, J. Guaschi, Lower central series of Artin-Tits and surface braid groups, J. Algebra 319 (2008), 1409-1427.

[3] J. S. Birman, Braids, Links, and Mapping Class Groups, Ann. Math. Stud. 82, Princeton University Press (1974).

[4] K. Borsuk, Drei Sätze über die n-dimensionale Euklidische Sphäre, Fund. Math. 20 (1933), 177-190.

[5] E. Fadell, S. Husseini, The Nielsen number on surfaces, Contemp. Math. 21 (1983), 59-99.

[6] E. Fadell, L. Neuwirth, Configuration Spaces, Math. Scand. 10 (1962), 111-118.

[7] D. L. Gonçalves, The Borsuk-Ulam theorem for surfaces, Quaest. Math. 29 (2006), 117-123.

[8] D. L. Gonçalves, J. Guaschi, The Borsuk-Ulam theorem for maps into a surface, Top. Appl. 157 (2010), $1742-1759$.

[9] D. L. Gonçalves, C. Hayat, P. Zvengrowski, The Borsuk-Ulam theorem for manifolds, with applications to dimensions two and three, Proceedings of the International Conference, Bratislava Topology Symposium, Group Actions and Homogeneous Spaces , (2010), 1-12.

[10] D. L. Gonçalves, M. R. Kelly, Wecken type problems for self-maps of the Klein bottle, Fixed Point Theory Appl. (2006), 1-15.

[11] V. C. Laass, A propriedade de Borsuk-Ulam para funções entre superfícies, Ph.D Thesis, IME, Universidade de São Paulo (2015).

[12] W. Magnus, A. Karrass, D. Solitar, Combinatorial Group Theory: Presentations of groups in terms of generators and relations, 2nd edition, Dover Publications, Inc., Mineola, NY, (2004).

[13] J. Matoušek, Using the Borsuk-Ulam Theorem, Universitext, Springer-Verlag (2002).

[14] K. Murasugi, B. I. Kurpita, A Study of Braids, Mathematics and Its Applications 484, Kluwer Academic Publishers, (1999).

[15] J. Nielsen, Untersuchungen zur Topologie der geschlossenen zweiseitigen Flächen, Acta Math. 50 (1927), 189-358.

[16] J. W. Vick, Homology Theory: An Introduction to Algebraic Topology, Graduate Texts in Mathematics 145, Springer-Verlag (1994).

[17] G. W. Whitehead, Elements of homotopy theory, Graduate Texts in Mathematics 61, Springer-Verlag (1978).

[18] H. Zieschang, E. Vogt, H.-D. Coldewey, Surfaces and Planar Discontinuous Groups, Lecture Notes in Mathematics 835, Springer-Verlag (1980). 